\title{
Global Intracellular Slow-Wave Dynamics of the Thalamocortical System
}

\author{
Maxim Sheroziya ${ }^{1,2}$ and Igor Timofeev ${ }^{1,2}$ \\ ${ }^{1}$ Department of Psychiatry and Neuroscience, Université Laval, Québec G1J 2G3, Canada, and ${ }^{2}$ Centre de recherche de l'Institut universitaire en santé \\ mentale de Québec, Université Laval, Québec G1J 2G3, Canada
}

It is widely accepted that corticothalamic neurons recruit the thalamus in slow oscillation, but global slow-wave thalamocortical dynamics have never been experimentally shown. We analyzed intracellular activities of neurons either from different cortical areas or from a variety of specific and nonspecific thalamic nuclei in relation to the phase of global EEG signal in ketamine-xylazine anesthetized mice. We found that, on average, slow-wave active states started off within frontal cortical areas as well as higher-order and intralaminar thalamus (posterior and parafascicular nuclei) simultaneously. Then, the leading edge of active states propagated in the anteroposterior/ lateral direction over the cortex at $\sim 40 \mathrm{~mm} / \mathrm{s}$. The latest structure we recorded within the slow-wave cycle was the anterior thalamus, which followed active states of the retrosplenial cortex. Active states from different cortical areas tended to terminate simultaneously. Sensory thalamic ventral posterior medial and lateral geniculate nuclei followed cortical active states with major inhibitory and weak tonic-like "modulator" EPSPs. In these nuclei, sharp-rising, large-amplitude EPSPs (“drivers") were not modulated by cortical slow waves, suggesting their origin in ascending pathways. The thalamic active states in other investigated nuclei were composed of depolarization: some revealing "driver"- and "modulator"-like EPSPs, others showing "modulator"-like EPSPs only. We conclude that sensory thalamic nuclei follow the propagating cortical waves, whereas neurons from higher-order thalamic nuclei display "hub dynamics" and thus may contribute to the generation of cortical slow waves.

Key words: cortex; dynamics; intracellular; slow oscillation; thalamus

\section{Introduction}

Slow waves are the hallmark of slow-wave sleep. They usually start from frontal and/or midline cortical areas and travel to lateral and posterior cortical areas (Massimini et al., 2004; Mohajerani et al., 2010; Nir et al., 2011; Stroh et al., 2013). Within the thalamocortical (TC) system, the slow-wave activity can be recorded from both cortex and thalamus (Contreras and Steriade, 1995). Slow waves originate in the neocortex as they can be recorded from isolated cortical structures (Sanchez-Vives and McCormick, 2000; Timofeev et al., 2000; Cossart et al., 2003), but not from the thalamus of decorticated animals (Timofeev and Steriade, 1996). During slow-wave activity, cortical neurons are active, depolarized, and fire spikes during local field potential (LFP) depth-negative waves, and they are silent and hyperpolarized during depth-positive LFP waves (Contreras and Steriade, 1995; Steriade et al., 2001; Timofeev et al., 2001; Chauvette et al.,

\footnotetext{
Received Oct. 18, 2013; revised May 15, 2014; accepted May 20, 2014.

Author contributions: M.S. and I.T. designed research; M.S. performed research; M.S. and I.T. analyzed data; M.S. and I.T. wrote the paper.

This work was supported by National Science and Engineering Research Council of Canada Grant 298475, Canadian Institutes of Health Research Grants MOP-37862 and MOP-67175, and National Institute of Neurological Disorders and Stroke Grants 1R01-NS060870 and 1R01-NS059740. We thank Serge Ftomov for technical assistance, Josée Seigneur for management assistance, and Sylvain Chauvette for comments on the manuscript.

The authors declare no competing financial interests.

Correspondence should be addressed to Dr. Igor Timofeev, Centre de recherche de l'Institut universitaire en santé mentale de Québec, 2601 de la Canardière Québec, Quebec G1J 2G3, Canada. E-mail: Igor.Timofeev@phs.ulaval.ca. DOI:10.1523/JNEUROSCI.4460-13.2014

Copyright $\odot 2014$ the authors $\quad 0270-6474 / 14 / 348875-19 \$ 15.00 / 0$
}

2011). Layer V pyramidal neurons play a leading role in the onset of active states (Sanchez-Vives and McCormick, 2000; Chauvette et al., 2010; Stroh et al., 2013). Intracellular recordings from ventral lateral and ventral posterior lateral thalamic nuclei demonstrated a passive hyperpolarization during cortical silent states and active inhibitory processes, originating from the reticular thalamic nucleus (RTN), during cortical active states (Contreras and Steriade, 1995; Contreras et al., 1996; Timofeev et al., 1996). Extracellular unit recordings from the ventrobasal complex and the posterior (PO) nucleus showed that these neurons fired at different phases of the slow oscillation (Slézia et al., 2011). Several studies demonstrate that the thalamus may actively contribute to the generation of slow-wave activity (Hughes et al., 2002; Crunelli and Hughes, 2010; David et al., 2013; Lemieux et al., 2014).

First-order thalamic nuclei receive inputs mainly from cortical layer VI and project into cortical layer IV and V/VI, whereas second-order thalamic nuclei receive inputs from cortical layer $\mathrm{V}$ and project into layer IV, superficial cortical layers, and into subcortical centers (Jones, 2009; Constantinople and Bruno, 2013). Axons from cortical layer VI (but not from layer V) send collaterals into the RTN (Deschênes et al., 1994; Bourassa et al., 1995). The inhibitory input into first-order thalamic nuclei is provided by the RTN, whereas higher-order thalamus receives inhibitory inputs mainly from the zona incerta (ZI) (Barthó et al., 2002; Trageser and Keller, 2004).

Large excitatory synapses (drivers) formed on proximal dendrites of first-order thalamic nuclei are formed by ascending ax- 
ons, whereas higher-order nuclei receive drivers originating mainly from layer V pyramidal neurons (Sherman and Guillery, 1996, 1998). A majority of modulatory inputs on TC neurons (small synapses formed on distal dendrites) are formed by corticothalamic axons.

Given the distinct patterns of connectivity, particularly in the origin of "driver" inputs and inhibitory inputs to different thalamic nuclei, we hypothesized that TC neurons from variable thalamic regions differently contribute to the cortical slow oscillation.

Here, using phase analysis for EEG and simultaneously recorded intracellular activities, we describe the global slow-wave dynamics of the TC system in ketamine-xylazine anesthetized mice that is the accepted model of slow-wave sleep (Sharma et al., 2010; Chauvette et al., 2011).

\section{Materials and Methods}

Experiments on anesthetized mice. Experiments were performed in accordance with the guideline of the Canadian Council on Animal Care and approved by the Universite Laval Committee on Ethics and Animal Research. Adult CD1 mice of both sexes were used in experiments. First, mice were anesthetized with ketamine and xylazine $(100$ and $10 \mathrm{mg} / \mathrm{kg}$, i.p.) and put in a stereotaxic frame. The scalp was infiltrated with lidocaine $(0.5 \%)$ before the skin incision. Two screws (stainless steel, $1 \mathrm{~mm}$ diameter) were inserted in the bone over the right somatosensory cortex (S1, lateral: $2.5 \mathrm{~mm}$; anteroposterior: $-1.5 \mathrm{~mm}$ from bregma; according to Paxinos and Franklin, 2001) and cerebellum (reference electrode) to monitor the EEG slow oscillation. Additional anesthesia was administrated every 30-40 min when EEG slow waves started weakening. The temperature of the platform in the stereotaxic frame where the mice were placed on was maintained at $37^{\circ} \mathrm{C}$. The gentle opening of the skull was done over the area of interest, and the dura was removed. Both cortical and thalamic slow-wave activity was very sensitive to cortical damage. For example, even light damage of the somatosensory cortex disrupted slow waves in ventral posterior medial (VPM) neurons. We found that it was practically impossible to remove the dura without cortical damage in mice. To avoid any contact of sharp tools with the cortex during removing the dura, we put a small drop of $3 \%$ peroxide on the dura. Peroxide itself did not penetrate under the dura but reacted with it. In 10-15 s, a bubble appeared under the dura. Then we washed out peroxide with saline and removed saline with mineral oil (Sigma). The space created by the bubble was used to remove the dura without a touch of the cortex. If detectable damage or bleeding from the cortex (not from the bone) were observed, obtained data were not used in phase analysis (see below). To avoid cortical surface drying, a mineral oil (Sigma) was applied over the exposed area. To reduce the brain pressure and pulsation, we opened the fourth ventricle. The stability of intracellular and LFP recordings was also reinforced by covering the opened cortical surface with $4 \%$ agar in saline. Since electrical shunting, $4 \%$ agar in saline was not used in experiments specifically investigating synchrony of LFP recorded from cortical surface. At the end of the recordings, the mice were killed by a lethal dose of sodium penthobarbital (120 mg/kg, i.p.) and perfused with 4\% PFA.

Recordings and labeling. AM 3000 amplifiers (A-M Systems) with custom modifications were used for EEG and LFP recordings (sampling rate $10 \mathrm{kHz}$ ). LFPs were recorded using monopolar tungsten electrodes (10-12 $\mathrm{M} \Omega$; FHC). In the majority of cases, the tips of LFP electrodes were located $0.8-0.9 \mathrm{~mm}$ from the cortical surface. The EEG recordings were obtained from a screw inserted in the bone. Intracellular and LFP recordings were made in the frontal association cortex (FrA, lateral: 1-1.5 $\mathrm{mm}$, anteroposterior: $2.5-2.6$ ), orbital (lateral: $1-1.5 \mathrm{~mm}$, anteroposterior: 2.5-2.6, $1.2 \mathrm{~mm}<$ depth $<3 \mathrm{~mm}$ ), motor (M1, lateral: $1.5-2 \mathrm{~mm}$, anteroposterior: $0.7-1.2 \mathrm{~mm}$ ), somatosensory (S1, lateral: $1-3 \mathrm{~mm}$, anteroposterior: -1 to $-2 \mathrm{~mm}$ ), visual ( $\mathrm{V} 1$, lateral: $2.5-3 \mathrm{~mm}$, anteroposterior: -2.5 to $-3 \mathrm{~mm}$ ), visual and retrosplenial (V-RS, lateral: $1-1.5$ $\mathrm{mm}$, anteroposterior: -3.5 to $-4 \mathrm{~mm}$ ), and the most posterior visual and retrosplenial (V-RS posterior, lateral: $3 \mathrm{~mm}$, anteroposterior: -4.5 to $-5 \mathrm{~mm}$ ) cortices. Intracellular recordings from the same cortical areas and from the thalamus were performed using glass micropipettes pulled from borosilicate glass capillaries (WPI; P-97, Sutter Instrument) and filled with a solution of $2 \mathrm{~m}$ potassium acetate $(50-70 \mathrm{M} \Omega)$. For recordings in the thalamus, the pipettes were also filled with $1 \%$ biocytin (Sigma). A high-impedance amplifier (Neurodata IR-283 amplifiers; Cygnus Technology) with active bridge circuitry was used to record the membrane potential and to inject current into the neurons. To stain thalamic cells, positive current pulse ( $200 \mathrm{~ms}$ on-off duty cycle, $0.5-1.5$ $\mathrm{nA}, 10 \mathrm{~min}$ ) was applied through the recording pipettes. After $1-2 \mathrm{~h}$ of survival, deeply anesthetized mice were perfused through the heart with saline followed by $4 \%$ PFA in $0.1 \mathrm{~m}$ phosphate buffer (PB). The brains were then removed and stored overnight in $4 \% \mathrm{PFA}$ in $\mathrm{PB}$ at $4^{\circ} \mathrm{C}$. Then $50-\mu \mathrm{m}$-thick frontal sections were cut.

During intracellular recordings from sensory thalamus, we also stimulated contralateral whiskers and hair on a mouse muzzle by air-puff or the contralateral eye by light flashes. To stimulate whiskers or muzzle, we used a small tube (diameter $1 \mathrm{~mm}$ ) connected to a compressed air balloon and equipped with a trigger. To stimulate an eye, we used white light-emitting diode directed into the contralateral eye also equipped with trigger.

Retrograde tracing. Mice were deeply anesthetized with isoflurane, placed in a stereotaxic frame as above, and a small opening was done over targeted thalamic nuclei. Glass micropipettes with a tip diameter 30-40 $\mu \mathrm{m}$ were filled with $0.2 \%$ cholera toxin subunit B (CtB, List Biological Laboratories); and using microinjector (Drummond Scientific), $9.2 \mathrm{nl}$ of $\mathrm{CtB}$ was delivered into the thalamus. $\mathrm{CtB}$ was injected either in VPM (lateral: $1.6 \mathrm{~mm}$, anteroposterior: -1.6 , depth: $3.2-3.3 \mathrm{~mm}$ ) or PO (lateral: $1.2-1.3 \mathrm{~mm}$, anteroposterior: -2.0 to $-2.2 \mathrm{~mm}$, depth: $3 \mathrm{~mm}$ ) thalamic nucleus. After the injection, the micropipettes were left inside for $15 \mathrm{~min}$ to reduce the leakage along the injection tract. The openings were then covered with dental cement, and the mice were placed into their home cages. After $7 \mathrm{~d}$ of survival, the mice were deeply anesthetized with ketamine and xylazine and perfused through the heart with saline followed by ice-cold 4\% PFA in PB. The brains were then removed and stored overnight in $4 \% \mathrm{PFA}$ in $\mathrm{PB}$ at $4^{\circ} \mathrm{C}$. Then, $50-\mu \mathrm{m}$-thick frontal sections were cut, and every other was collected.

Immunohistochemistry. To visualize biocytin-labeled cells, freefloating sections were incubated in ABC kit (1:200; Vector Laboratories) in TBS for $2 \mathrm{~h}$. After rinses, sections were incubated in TBS containing $0.05 \% \mathrm{DAB}$ (Sigma), $0.005 \% \mathrm{CoCl}_{2}$, and $0.00125 \% \mathrm{H}_{2} \mathrm{O}_{2}$ for $5-10 \mathrm{~min}$.

$\mathrm{CtB}$-containing sections were first incubated in $2 \%$ normal rabbit serum, $0.5 \% \mathrm{BSA}$, and 0.3 Triton $\mathrm{X}-100$ in PBS overnight at $4^{\circ} \mathrm{C}$. The incubation in goat anti-CtB (1:4000; List Biological Laboratories), $2 \%$ normal rabbit serum, $0.5 \% \mathrm{BSA}$, and 0.3 Triton in PBS for $4 \mathrm{~d}$ at $4^{\circ} \mathrm{C}$ followed. After rinses, sections were incubated in biotinylated rabbit anti-goat IgG (1:200; List Biological Laboratories) for $2 \mathrm{~h}$, and then ABC and $\mathrm{DAB}$ reaction followed like above. In the end, all sections were mounted onto gelatin-coated glass slides, dehydrated, and coverslipped. Microphotographs were taken, and Image Pro software (Media Cybernetics) was used for automatic quantification of the labeled cells (see below).

Electrophysiological data analysis. To detect phases of the slow oscillation, we used the Hilbert transformation (Fig. 1A) for which the EEG was filtered (bandpass frequency $0.2-4 \mathrm{~Hz}$ ). The extracted phase changes in a range of -1 to 1 of fraction of $\mathrm{Pi}$. The instantaneous phase \pm 1 corresponds to the middle of the negative peak of the cortical EEG, whereas the instantaneous phase 0 corresponds to the middle of the positive peak of the cortical EEG (Fig. 1A). For phase analysis, we extracted spikes and postsynaptic potentials/noise from simultaneously recorded intracellular activities. Only stable intracellular recordings (at least $1 \mathrm{~min}$ recordings, but usually $2-5 \mathrm{~min}$ ) were used for phase analysis (stable membrane potential, $<-60 \mathrm{mV}$ during silent states, spikes amplitude $>45 \mathrm{mV}$ ). Spikes were detected using a threshold. IPSPs (negative peaks) were detected manually. To extract EPSPs, the intracellular activity was filtered between 0 and $1000 \mathrm{~Hz}$, then differentiated, and the threshold was set on. To extract all EPSPs and/or synaptic noise, we used a threshold of 0.03 $0.05 \mathrm{~V} / \mathrm{s}$, whereas to extract large-amplitude EPSPs in the thalamus, we used a threshold of $0.1 \mathrm{~V} / \mathrm{s}$ (Fig. 1A). IPSPs were detected manually based on sharp negative deflection of $>5 \mathrm{mV}$ and their characteristic shape 
A

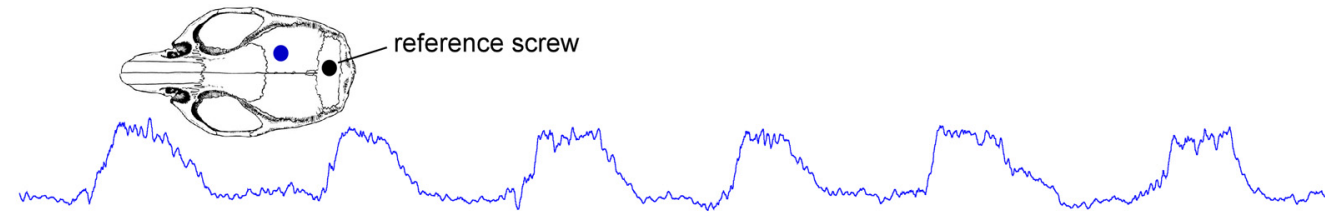

EEG S1

Filtered

EEG S1

+ Hilbert

transform

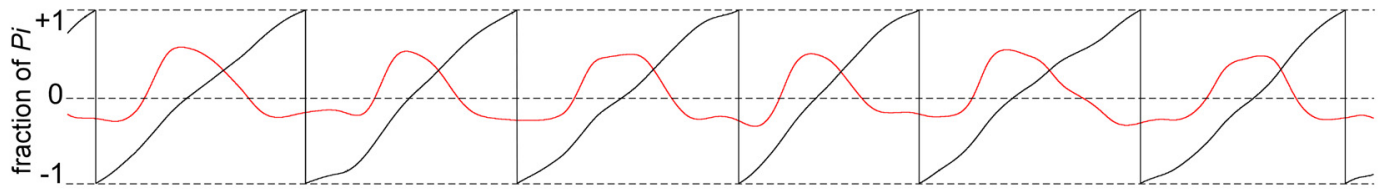

Vintra-cell

VPM thalamus

$\mathrm{d}\left(V_{\text {intra-cell }) / \mathrm{d} t}\right.$

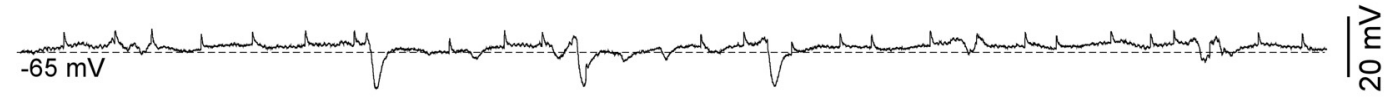

EPSPs

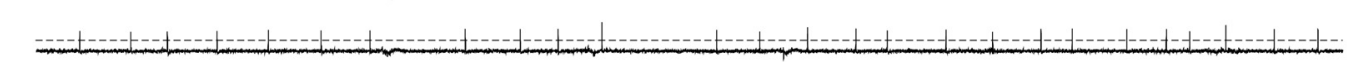




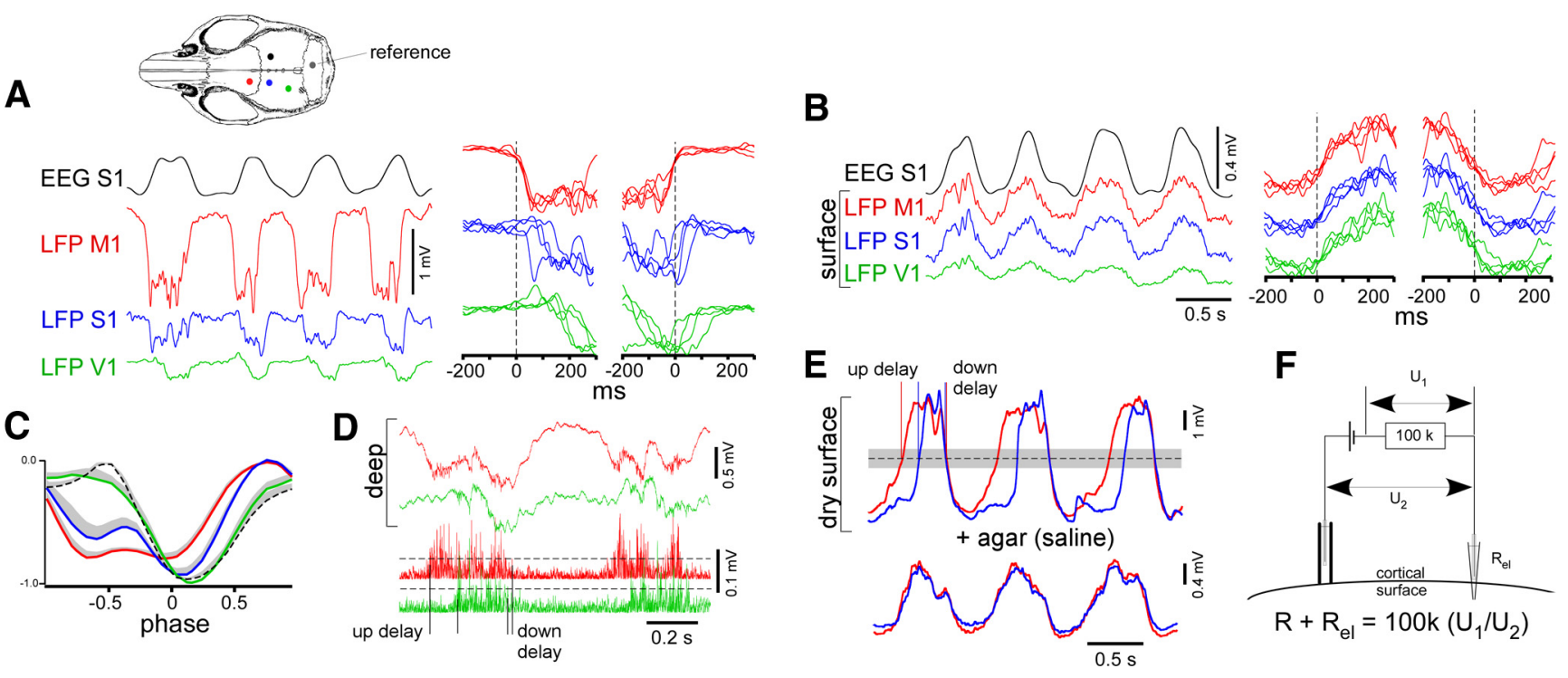

Figure 2. LFP/EEG recordings in the mouse cortex. $A$, Simultaneously recorded LFP and EEG activities in the motor, somatosensory, and visual cortex in deep layers (0.7-0.9 mm) as estimated from manipulator reading. The location of electrodes is schematically shown by colors on the mouse skull drawing. Left, Superposition of the beginning and the end of active states at a higher temporal resolution. $\boldsymbol{B}$, LFP activities recorded from the wet cortical surface at the same points as in A. C, Averaged LFP waves from deep layers plotted against phase. In each animal ( $n=6$ mice), 2 min segments of LFPs were recorded simultaneously by pairs from M1 and successively in $\mathrm{S1}$, and then V1, and RS (dotted line, the cortical area is shown by dotted point on the mouse skull drawing). Before averaging in a group, waves were normalized by maximum values. The positive part of SEM is shown with gray (negative part is not shown). $D$, An example of paired LFPs from deep layers in M1 and V1 and lower the corresponding absolute values of $\gamma(50-300 \mathrm{~Hz})$ activities are shown. The delays between $\gamma \mathrm{LFP}$ signals were detected using the crossings with the thresholds $(7$ SD of silent state activities). $\boldsymbol{E}$, An example of paired LFPs from dry cortical surface in M1 and S1 before and after (bottom traces) of agar application on the cortex. The delays between LFP signals were detected using the crossings with half of wave amplitude. On average, the delays were insensitive to the threshold amplitude within significant range (shown with gray). $\boldsymbol{F}$, The circuit that was used to measure a passive impedance of the cortex. The pipettes were filled with $3 \mathrm{~m} \mathrm{NaCl}$ in $4 \%$ agar to avoid leakage, and chlorinated silver wires were used inside the pipette. Sinusoidal potential was applied for measurements $(2 \mathrm{~Hz}$, amplitude $0.4 \mathrm{~V})$.

intracellular wave itself. We also calculated the first derivatives of the intracellular waves and EPSP histograms by phase and their mean vectors (see below). Before differentiation, waves and histograms plotted against phase were smoothed with a binomial filter.

Perievent time histograms were also plotted for extracted postsynaptic potentials. To quantify rising and descending slopes of the histograms, we measured the times (phases) and amplitudes of the histograms at their half amplitude and additionally at one-tenth amplitude for decay measured from minimum (see Figs. 9 and 11).

To reveal the delays between simultaneous LFP and intracellular recordings, the crossings at half of amplitude within each slow-wave cycle were detected (Fig. $2 E$ ). To analyze delays between LFP $\gamma$ activities, we calculated their absolute values $\left(\gamma^{2}\right)^{1 / 2}$, and then the crossings with the threshold (7 SD of silent periods) corresponding to the transitions between active/silent states were detected (Fig. 2D).

Wave analysis and circular statistics were performed with Igor Pro version 6.2 software (WaveMetrics). Basic statistical and other data analysis was performed using Sigma Plot (Systat Software). Data are expressed as mean $\pm \mathrm{SD}$. or SEM when indicated. If the normality and equal variance tests passed $(p>0.05)$, then a two-tailed (parametric) $t$ test was used; otherwise, a Mann-Whitney sum rank test (nonparametric) was used (significance level, $p<0.05$ ).

\section{Results}

\section{Slow-wave propagation over the cortex}

We recorded slow-wave activity with monopolar LFP electrodes either simultaneously from the left M1, S1, V1, and V-RS cortices (Fig. 2A) or in pairs. In all experiments, EEG recorded from the contralateral somatosensory cortex was used for Hilbert transformation. Time and phase shifts between slow waves from M1, S1, V1, and V-RS cortices were apparent when LFP activities were recorded in deep layers (depth $0.7-0.9 \mathrm{~mm}, n=6$ animals; Fig. $2 A, C)$. When LFP activities were recorded from the wet cortical surface, no apparent shifts between slow waves from M1, S1, and V1 cortical areas were found (Fig. $2 B ; n=3$ animals). The same result was obtained with EEG screws inserted in the skull on these cortical areas ( $n=3$ animals; data not shown). However, if LFP activities were recorded from the dry cortical surface, positive large-amplitude $(4.5 \pm 1 \mathrm{mV})$ propagating signals were detected $(n=3$ animals; Fig. $2 E)$. The active states clearly tended to terminate simultaneously within different cortical areas. Application of agar (based on saline) on the cortical surface diminished the amplitude of LFP signal $(1.1 \pm 0.2 \mathrm{mV})$ as well as eliminated the delays in the onset of active states (Fig. 2E). Because $\gamma$ activity is a proven indicator of active states (Mukovski et al., 2007), we calculated the absolute values of LFP $\gamma(50-300 \mathrm{~Hz})$ activities recorded either from the surface or deep layers (Fig. 2D). Then we detected delays between the onsets of active or silent states (up delays or down delays correspondingly) as shown in Figure 2D, E between slow waves from different cortical areas and plotted delay as histograms (Fig. 3). We also plotted averaged LFP and absolute value of $\gamma$ LFP activities against phase (Fig. 3). EEG and LFP of wet cortical surface (Fig. $3 A, B$ ) did not show propagation of slow-wave activity over the investigated cortical hemisphere. Normalized and plotted against phase, the LFP waves from wet cortical surface also coincided with the EEG wave from the contralateral hemisphere (Fig. 3B1). LFP slow wave from dry surface and $\gamma$ LFP activities from dry surface or from deep layers revealed propagation of the leading edge of the surface positive (active state) wave in the anteroposterior direction and simultaneous termination on average of the wave (Fig. 3C-E). The $\gamma$ LFP signal recorded from depth had higher amplitude compared with surface $\gamma$ signal (Fig. 3D1,E1). Generally, slow waves started from the $\mathrm{M} 1$ area, propagated to $\mathrm{S} 1$, and then to $\mathrm{V} 1$ and $\mathrm{V}$-RS areas $(n=6$ animals; Fig. 3E1). The distributions of up delays (Fig. 3E2) revealed fluctuations of anteroposterior propagation (probability $30 \pm 18 \%$ from $S 1$ into M1, $8 \pm 7 \%$ from V1 to M1, $3 \pm 3 \%$ from 
A1

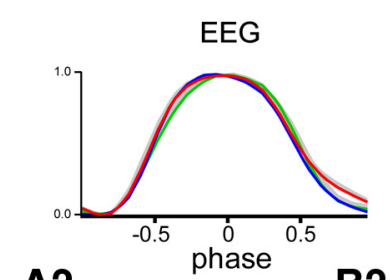

A2

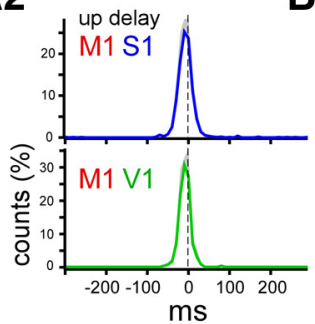

B2

B1
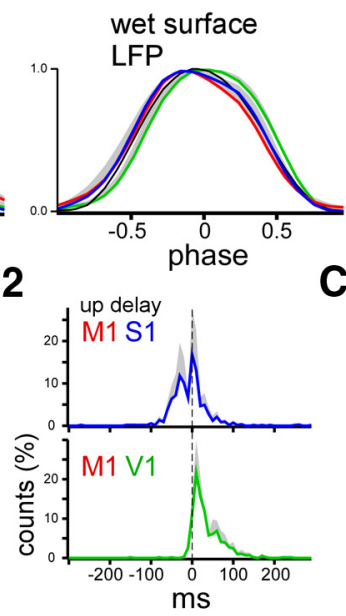

A3

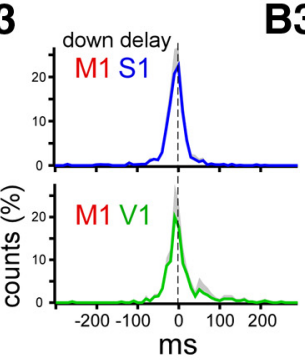

B3

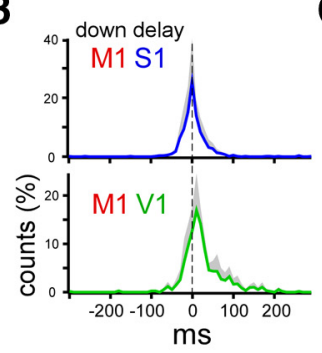

C1

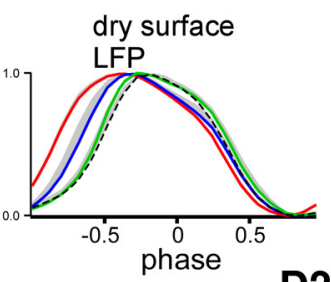

C2

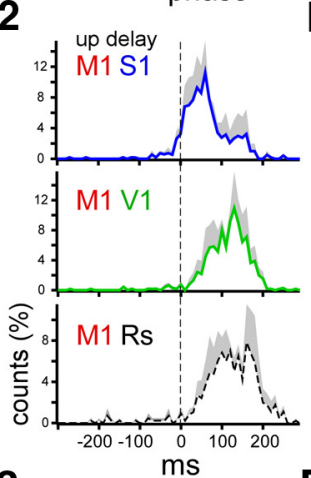

C3

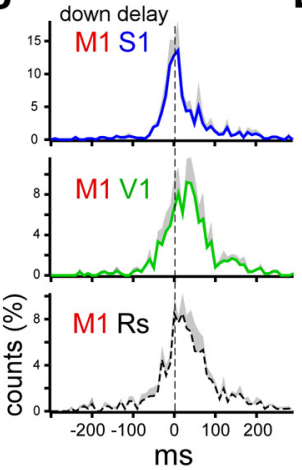

D1

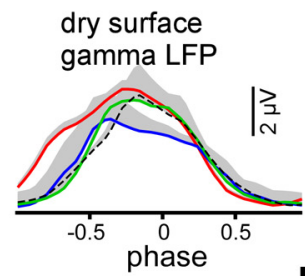

D2
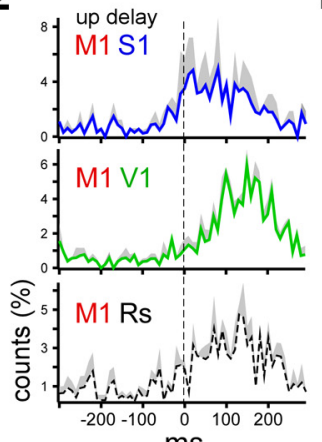

D3
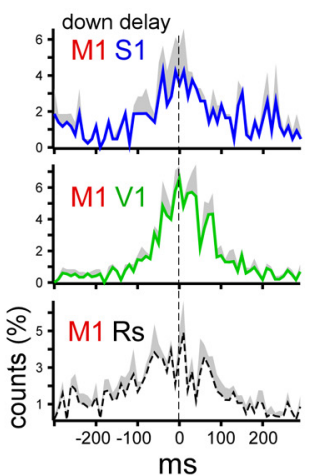

E1

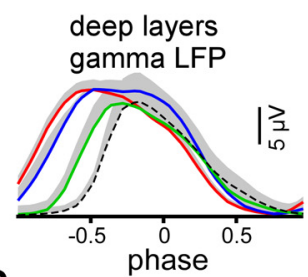

E2

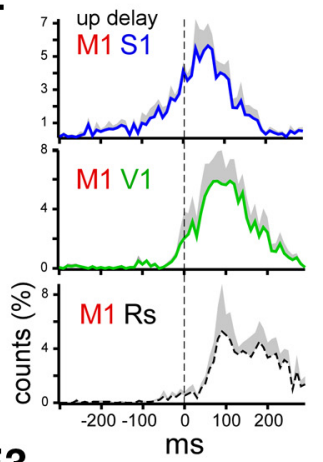

E3

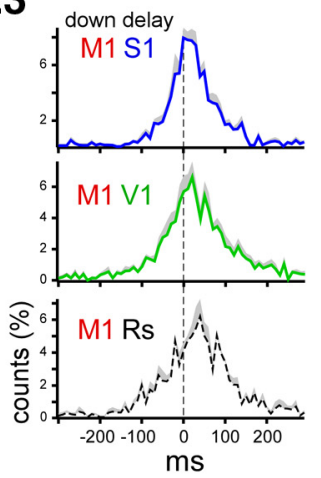

Figure 3. Propagation and termination of cortical LFP/EEG activities. Activities were recorded simultaneously by pairs in M1 and successively in S1, and then V1, and RS (color code as in Fig. 2). $A$, EEG activities recorded with screws were coherent across $\mathrm{M1}$ 1, $\mathrm{S}$, and $\mathrm{V} 1$. $A 1$, Averaged EEG signals from $\mathrm{M} 1, \mathrm{S1}$, and V1 are plotted against phase ( $n=3$ animals). Before grand averaging, waves were normalized by maximum values. The histograms for up (A2) and down (A3) M1-S1 and M1-V1 delays of the same EEG recordings are shown. The delays were detected as shown in Figure $2 E$. When S1 preceded M1 (V1 preceded M1), the time intervals calculated as negative. B, The similar plots for LFPs of wet cortical surface ( $n=3$ animals). B1, Black line indicates contralateral EEG. Other conventions as in $\boldsymbol{A} \mathbf{2}$ and $\boldsymbol{A}$ 3. C, The similar plots for LFPs of dry cortical surface are shown ( $n=3$ animals). Other conventions as in $\boldsymbol{A} \mathbf{1}$ and $\boldsymbol{A}$ 3. D, Plotted against phase, the absolute values of $\gamma$ LFP signals (D1) recorded from the dry cortical surface, and up (D2) and down (D3) delay histograms are shown ( $n=3$ animals). Other conventions as in $\boldsymbol{A}$ and $\boldsymbol{A 3}$. The delays were detected as in Figure 2D.E, The similar plots for the absolute value of $\gamma$ LFP signals recorded from the deep layers ( $n=6$ animals). In all panels, the positive part of SEM is shown with gray (negative part is not shown).

V-RS to M1, $n=6$ animals; Fig. 3E2). Although the probabilities of posterior-anterior propagation obtained from largeamplitude LFP signal of the dry surface was lower (probability $10 \pm 8 \%$ from S1 into M1, $2 \%-3 \%$ from M1 to V1 and V-RS, $n=$ 3 animals; Fig. 3C2). The mean up M1-S1, M1-V1, and M1 (VRS) delays obtained from $\gamma$ LFP signal recorded in deep layers were $48 \pm 45,98 \pm 46$, and $147 \pm 42 \mathrm{~ms}$, respectively (up M1-S1 vs up M1-V1, $p=0.054$; up M1-S1 vs up M1-(V-RS), $p=0.003$ ). The mean down M1-S1, M1-V1, and M1-(V-RS) delays were $14 \pm 12,24 \pm 19$, and $28 \pm 14 \mathrm{~ms}$, respectively (down M1-S1 vs down M1-(V-RS), $p=0.1$ ). Taking into account a large scatter of down delays (almost $\pm 200 \mathrm{~ms}$ centered around $0 \mathrm{~ms}$; Fig. 3E3) compared with the relatively small $(\sim 20 \mathrm{~ms})$ and insignificant differences in mean down delays, we conclude that, in the slowwave time scale, the active states tended to terminate simultaneously within different cortical areas.

We did not detect neuronal correlations, which could explain phase and time shifts between wet surface and deep LFP slowwave signals in mice (Fig. $4 H$ ). The simplest explanation for low variability of surface EEG (Fig. $3 A$ ) and LFP recorded from the cortical surface covered with liquid or agar (Fig. $3 B$ ) is a shunting effect, which leads to a spreading of the extracellular potential along the cortical surface compared with deep layers. To test this possibility, we measured the impedance between two pipettes, one of which was located on the wet cortical surface and the other was either on the surface or in deep layers (Fig. $2 F$ ). The impedance of the pipettes themselves was $190 \pm 50 \mathrm{k} \Omega$. When the second pipette was on the cortical surface and another one was inserted into the depth, the impedance of cortical tissue was $43 \pm$ $25 \mathrm{k} \Omega$ ( $n=5$ pipette pairs). We failed to get a satisfactory estimation for the impedance of tissue on the surface and any distance dependence apparently because of pulsation of the cortex (measured impedance was $1 \pm 7 \mathrm{k} \Omega$ ). For comparison, the impedance between the same pipettes connected with salinemoistened napkin $5 \times 10 \mathrm{~mm}$ was $6.5 \pm 1.3 \mathrm{k} \Omega$. These results indicate that the surface LFP signal depends on experimental conditions, whereas EEG signals recorded from screws do not represent the traveling wave but are reflecting the large-scale brain activity and therefore provide a reliable reference for the slow-wave phase detection. 
A
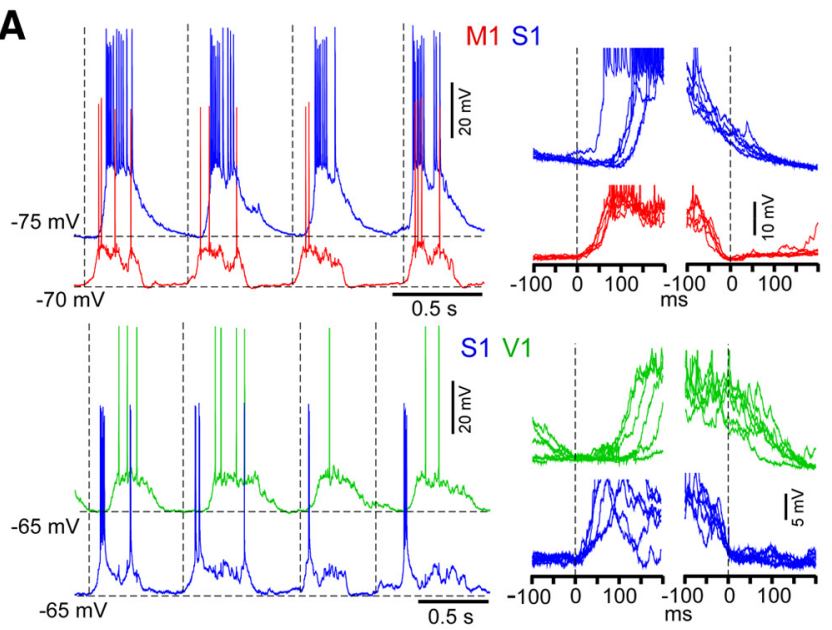

C

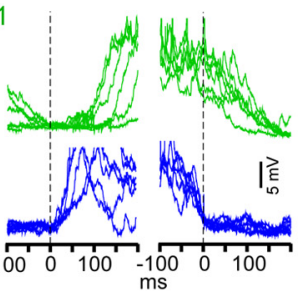

D
B

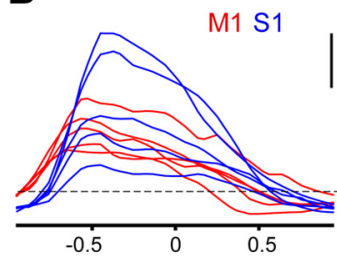

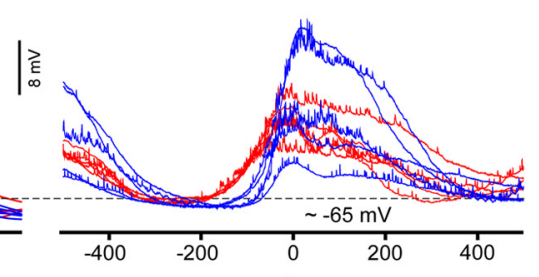
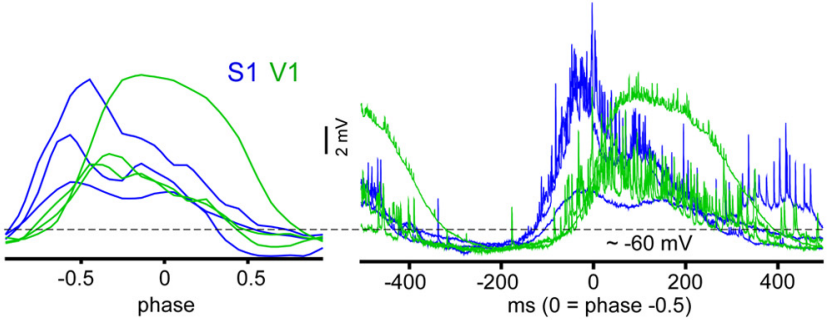

E
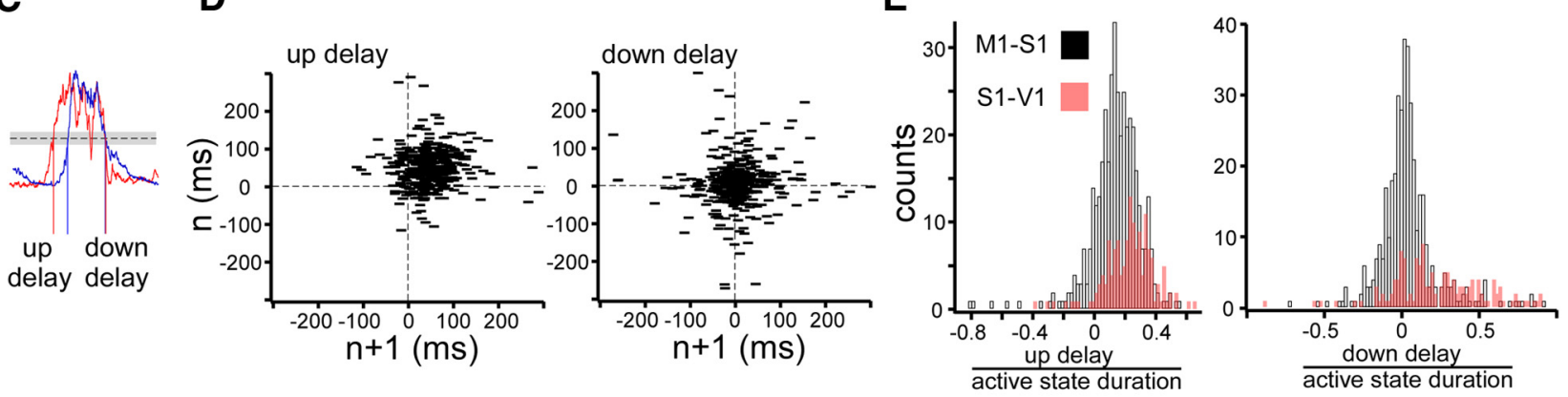

$\mathbf{F}$
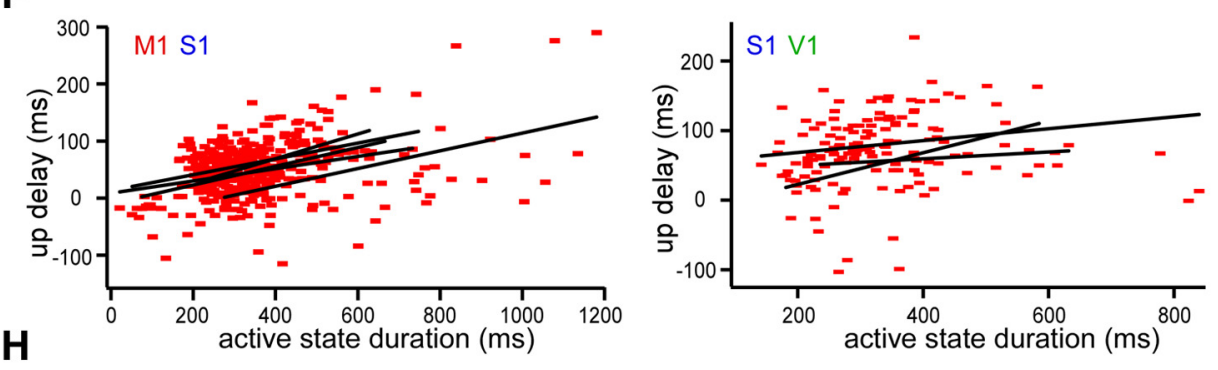

G
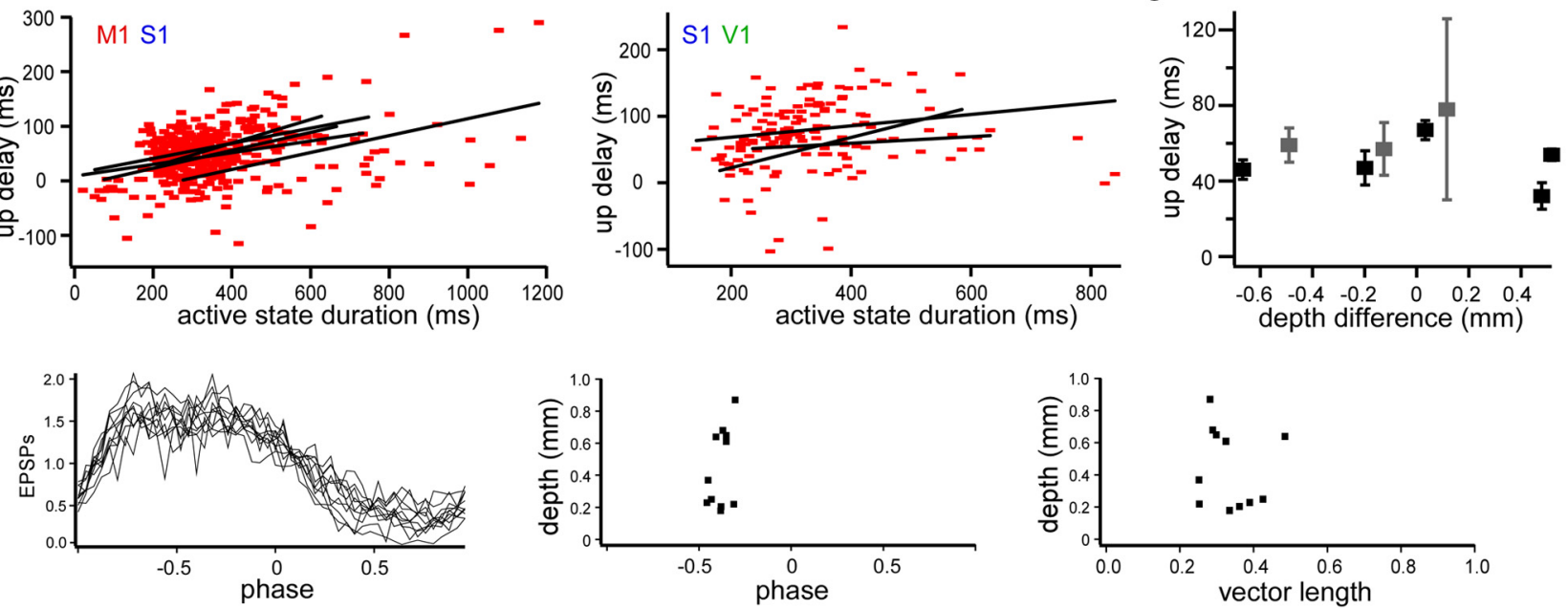

Figure 4. Paired intracellular recordings from different cortical areas. $A$, The examples of simultaneously recorded intracellular activities of two neurons from the motor and somatosensory, and from the somatosensory and visual cortices. The cortical locations are indicated by colors. Note the time delays between active state onsets in the cell pairs. $\boldsymbol{B}$, Superposition of averaged membrane potentials of $n=5 \mathrm{M} 1-\mathrm{S1}$ and $3 \mathrm{S1-V1}$ neuronal pairs are plotted against phase (left) and time (right). In the time plots, zero time corresponds to the instantaneous phase -0.5 . C, The methodological approach for the delay analysis of paired intracellular recordings. The crossings at half of amplitude within each slow-wave cycle were detected. The delays were almost insensitive to the threshold amplitude within significant range (shown with gray). The time intervals between crossings were calculated and represent up and down delays as shown. When S1 preceded M1 (V1 preceded S1), the time intervals calculated as negative. Duration of active state calculated as the time interval between down and up crossings. D, Superposition of all M1-S1 up and down delays of 5 neuronal pairs ( $n$ delay plotted against $n+1$ delay). $\boldsymbol{E}$, Distribution of all M1-S1 (shown with black) and S1-V1 (shown with pink) up (left) and down (right) delays divided by the corresponding durations of $\mathrm{M} 1$ and $\mathrm{S} 1$ active states, respectively. $\boldsymbol{F}, \mathrm{All}$ M1-S1 and $\mathrm{S1-V1}$ up delays plotted against the duration of $\mathrm{M} 1$ and $\mathrm{S} 1$ active states, respectively. Black lines indicate linear fit for each neuronal pair. $\mathbf{G}$, The mean up delays between neuronal pairs plotted against depth difference of neurons. The depth differences were calculated as the difference between $\mathrm{M} 1$ and $\mathrm{S1}$ neurons (between $\mathrm{S} 1$ and V1, shown with gray). $\boldsymbol{H}$, Left, Superposition of EPSP phase histograms normalized by mean representing the intracellular activity of 11 neurons recorded in the frontal cortex at different depths. Middle, Right, Mean vector phases and lengths calculated from the same EPSP histograms plotted against depth from which the neurons were recorded.

To average intracellular activities from different cortical and subcortical structures obtained in independent experiments, the most integrative and stereotypic process should be taken as a reference wave. This process should not be influenced by local fluctuations, and it should reflect as much as possible an averaged large-scale brain activity. In previous studies, to eliminate the influence of time variability of slow-wave duration, filtered EEG was taken for phase analysis with Hilbert transform (Mallet et al., 
2008; Slézia et al., 2011). Because the delays of propagation of EEG were minimal (Fig. $3 A$ ), in the remaining part of the study, we used this signal as reference to align intracellular recordings obtained in different cortical or thalamic locations.

\section{Paired intracellular recordings from different cortical areas} To investigate the cellular basis of the results obtained from LFP activities, we performed dual intracellular recordings of neuronal activities from M1 and S1 ( $n=5$ pairs of neurons) and from $\mathrm{S} 1$ and V1 ( $n=3$ pairs of neurons) cortices. In all recorded pairs of neurons, a majority of $\mathrm{M} 1$ active states started off before $\mathrm{S} 1$ active states, whereas active states in $\mathrm{S} 1$ also started before V1. Examples are shown in Figure $4 A$. Using Hilbert transform for simultaneously recorded EEG activities, we extracted the phase for each recording and plotted membrane potentials from pairs of neurons against the phase (Fig. $4 B$, left) and time (Fig. $4 B$, right). In time plots, zero time corresponded to the instantaneous phase -0.5 . Averaged membrane potentials on phase and time plots revealed phase and time delays between neurons from different cortical areas. The mean time delays (measured at half rising times; Fig. 4B, right) in M1-S1 and S1-V1 neuronal pairs were $46 \pm 11 \mathrm{~ms}$ and $55 \pm 25 \mathrm{~ms}$, respectively. Considering the mean distance from $\mathrm{M} 1$ to $\mathrm{S} 1$ and from $\mathrm{S} 1$ to $\mathrm{V} 1$ as $\sim 2 \mathrm{~mm}$, the slowwave propagation velocity was $40 \pm 13 \mathrm{~mm} / \mathrm{s}$, which was similar to the velocity previously described in other studies (Mohajerani et al., 2010; Ruiz-Mejias et al., 2011).

To show the variability of the slow-wave propagation, we also calculated up and down delays between individual active states of simultaneously recorded neurons (Fig. 4C). Up and down delays fluctuated around the mean values and did not reveal any temporal/serial dependencies (Fig. 4D). The probabilities of posterior-anterior propagation were $14.5 \%$ (from $6 \%$ to $25 \%$ ) and $9 \%$ (from $3 \%$ to $13 \%$ ) in $\mathrm{M} 1-\mathrm{S} 1$ and $\mathrm{S} 1-\mathrm{V} 1$ pairs of neurons correspondingly. As in LFP experiments, the intracellular active states tended to terminate simultaneously (except one V1 neuron; Fig. $4 B$ ). Similar to a previous study on cats (Volgushev et al., 2006), individual down delays revealed large fluctuations centered around $0 \mathrm{~ms}$ (Fig. $4 D, E$ ). The mean values of SEM for up and down delays in M1-S1 pairs were 5.74 and $6.02 \mathrm{~ms}$, respectively (the extent of fluctuations was similar). We did not detect any apparent correlation between the depth of neurons, the time delays between them, and the preferential phase of involvement of individual neurons in the slow oscillation (Fig. $4 G, H$ ). In all eight pairs of neurons recorded in this study, we found a positive correlation between the up delays and the durations of active states (Fig. $4 F$ ).

\section{Intracellular slow-wave patterns in the mouse cortex and thalamus}

To investigate how cortical and thalamocortical cells of different locations are involved in slow oscillation, we performed intracellular recordings within six cortical areas along the anterocaudal direction (see Materials and Methods) and from specific and nonspecific thalamic nuclei. Together, we recorded 18, 8, 11, 5, 7, and 7 neurons from FrA-O, M1, S1, V1, V-RS, and V-RS posterior cortical areas and 159 cells in the thalamus. Thalamocortical cells were identified either by their ability to respond with a characteristic low-threshold spike (LTS) in response to a hyperpolarizing current pulse or by morphological location. Within VPM nucleus and lateral geniculate nucleus (LGN), neurons were also usually identified by their response to sensory stimulation (whiskers and hair: air puff and eye-light flash, respectively).

As expected, all cortical neurons demonstrated an alternation of depolarized active (UP) and hyperpolarized silent (DOWN) states, whereas thalamic neurons revealed two distinct slow-wave patterns. Eighty-four thalamic neurons displayed unitary and/or spindle-like IPSPs, which followed cortical slow waves. The other 75 thalamic neurons displayed depolarized active states, which accompanied cortical slow waves, although occasionally we could also identify IPSPs. The examples of EEG and the two characteristic intracellular slow-wave patterns of TC neurons recorded simultaneously with cortical S1 neurons are shown in Figure 5.

Within particular thalamic nuclei, we identified the location of 51 neurons (Table 1; Figs. 5 and 6). All the neurons from the higherorder nuclei we identified histologically. Neurons from sensory nuclei were identified either by their response to sensory stimulation or both histologically and by their response to sensory stimuli. The neurons within VPM, LGN, PO, and AV nuclei generated LTS in response to a hyperpolarizing current pulse, whereas the neurons from PF, central lateral (CL), and AD did not generate LTS. Usually, we did not observe spontaneous LTS in the mouse's thalamus (Figs. 5 and 6), and only some PO neurons displayed spontaneous LTS when steady hyperpolarizing current was intracellularly injected (data not shown). Intralaminar PF neurons displayed the lowest input resistance (Table 1), which was significantly different from the input resistance of PO neurons $(16 \pm 5 \mathrm{M} \Omega$ vs $25 \pm 6 \mathrm{M} \Omega$, respectively, $p=0.005)$, of AV neurons $(24 \pm 8 \mathrm{M} \Omega, p<0.05)$, and VPM and LGN neurons together $(23 \pm 7 \mathrm{~m} \Omega, p<0.05)$.

Using interpolation, we plotted membrane potentials against phase for all stable intracellular cortical and thalamic recordings (Fig. 7). At least 1 min segments of recordings with the robust slow oscillation of well-anesthetized mice were used. On average, the neuronal membrane potentials from PO ( $n=7$ neurons), parafascicular (PF, $n=6$ neurons), CL ( $n=1$ neuron, data not shown), anterior dorsal (AD, $n=1$ neuron; data not shown), and anterior ventral (AV, $n=7$ neurons) nuclei displayed depolarized waves (averaged active states), which accompanied cortical depolarized waves (Fig. 7). In 3 of 7 analyzed PO cells, we also recorded occasional IPSPs that produced a local minimum during active states (Fig. 7, red dotted line). The averaged intracellular slow waves within VPM $(n=14$ neurons) and LGN $(n=6$ neurons) nuclei were dominated by inhibitory activities that produced averaged IPSPs with negative peaks $<-65 \mathrm{mV}$. The membrane potential depolarization of all cortical cells as well as from all the neurons within thalamic PO, PF, CL, AD, and AV nuclei were phase-locked ( $p<0.05$, Moore-Rayleigh test).

\section{Synaptic activities during slow oscillations in the mouse thalamus and cortex}

The main excitatory inputs formed on thalamocortical neurons are usually divided to drivers and modulators based on the EPSP amplitude and rising time (Li et al., 2003; Reichova and Sherman, 2004). To our knowledge, no driver-like EPSPs were reported in the cortex. Typical examples of slow-wave cycles from different thalamic nuclei and from the somatosensory cortex are shown in Figure 8A. Large-amplitude, well-defined unitary EPSPs were a common feature of spontaneous activity of all the neurons recorded within PO, AV, VPM, and LGN nuclei (Fig. 8A, red arrows). Sensory responses within projecting VPM and LGN neurons were also composed of large-amplitude, well-defined unitary EPSPs as well IPSPs (Fig. 6B). Small-amplitude EPSPs occurring in groups were present in neurons from all recorded nuclei (Fig. $8 A$, blue arrows), but within projecting VPM and LGN nuclei they were weak, even if the membrane potential was hyperpolarized with injected current. We consider EPSPs called here "large-" and "small-" amplitude corresponding to thalamic drivers and modulators, respectively. We did not detect large- 

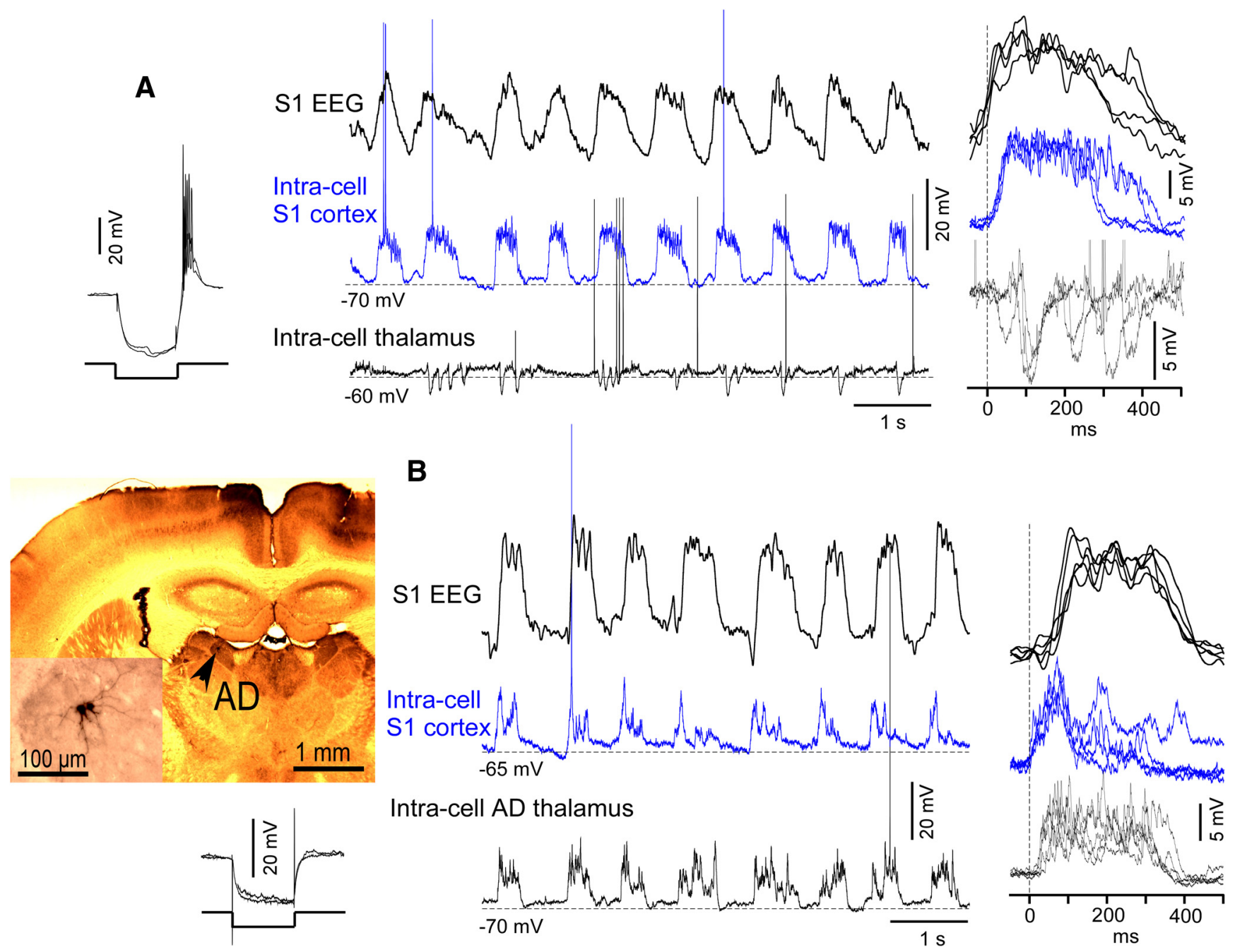

Figure 5. Two intracellular slow-wave patterns recorded from the mouse thalamus. $A$, Simultaneously recorded intracellular activities of thalamic and cortical $S 1$ neurons and contralateral EEG during the slow oscillation in anesthetized mice. Left, The thalamic neuron reveals a characteristic LTS in response to a hyperpolarizing ( $100 \mathrm{~ms}, 0.5 \mathrm{nA})$ current pulse. During cortical depolarizing active states, the TC neuron displayed unitary or repetitive IPSPs. Thalamic IPSPs follow the onset of cortical active states. $\boldsymbol{B}$, A segment of EEG and intracellular activities recorded from another pair of thalamic (AD) and cortical neurons. A morphologically identified thalamic neuron (left) did not generate LTS in response to a hyperpolarizing current pulse (inset) of different amplitude (data not shown). The thalamic neuron generated active states composed of multiple depolarizing events that followed cortical active states. There is a time delay between the onset of intracellular cortical and thalamic active states (right).

Table 1. Histologically identified neurons within the thalamus ${ }^{a}$

\begin{tabular}{lllll}
\hline $\begin{array}{l}\text { Thalamic } \\
\text { nucleus }\end{array}$ & $\begin{array}{l}\text { Number of } \\
\text { neurons }\end{array}$ & Slow-wave pattern & $\begin{array}{l}\text { Input } \\
\text { resistance, M } \Omega\end{array}$ & $\begin{array}{l}\text { LTS in response to } \\
\text { hyperpolarizing pulse }\end{array}$ \\
\hline VPM & 15 & IPSPs & $23 \pm 7$ & + \\
LGN & 7 & IPSPs & $23 \pm 7$ & + \\
P0 & 11 & EPSPs, active states & $25 \pm 6$ & + \\
PF & 6 & EPSPs, active states & $16 \pm 5$ & - \\
CL & 1 & EPSPs, active states & - & - \\
AD & 1 & EPSPs, active states & - & - \\
AV & 10 & EPSPs, active states & $24 \pm 8$ & + \\
\hline
\end{tabular}

${ }^{a}$ The neuronal location was identified according to Paxinos and Franklin (2001): AV, Anteroventral thalamic nucleus; $A D$, anterodorsal thalamic nucleus; $\mathrm{CL}$, centrolateral thalamic nucleus; $\mathrm{LGN}$, lateral geniculate nucleus; $\mathrm{PF}$, parafascicular thalamic nucleus; $\mathrm{PO}$, posterior thalamic nuclei group; VPM, ventral posteromedial thalamic nucleus.

amplitude EPSPs in cortical neurons (Fig. 8A), so all cortical EPSPs displayed modulator-like behavior.

First, we compared mean amplitudes and kinetics (half rise and half decay time counting from the EPSP peak; Fig. $8 B$ ) of the large-amplitude EPSPs within VPM, LGN, AV, and PO $(n=9,7$, 10 , and 11 neurons, respectively). All measurements were performed without injection of intracellular steady current and with a membrane potential of $\sim-60$ to $-75 \mathrm{mV}$. We found that EPSP amplitude within PO neurons was significantly higher than within VPM, LGN, and AV $(7.37 \pm 0.45 \mathrm{mV}$ vs $4.15 \pm 0.25$, $4.32 \pm 0.25$, and $4.72 \pm 0.47 \mathrm{mV}$, respectively, mean $\pm \mathrm{SEM}, p<$ 0.001 ; Fig. $8 B$ ). Both rising and decaying phases of EPSPs within VPM, LGN, and AV nuclei were significantly $(p<0.05)$ faster than they were within second-order PO neurons (Fig. 8B). "All or none" behavior of driver EPSPs was reported previously (Li et al., 2003; Reichova and Sherman, 2004; Groh et al., 2008); therefore, we also calculated the ratio (SD)/(mean amplitude) for the largeamplitude EPSPs extracted in each neuron (an "all or none" behavior supposing an $\mathrm{SD} /$ mean ratio $\ll 1$ ). We found that the ratio $\mathrm{SD} /$ mean amplitude was significantly smaller in VPM neurons compared with LGN, AV, and PO neurons $(0.153 \pm 0.038$ vs $0.322 \pm$ $0.126,0.233 \pm 0.032$, and $0.397 \pm 0.13$, respectively, $p \leq 0.001)$. The ratios for $\mathrm{AV}$ and $\mathrm{PO}$ neurons were significantly different, too $(p=0.007)$. There were no evidently pronounced largeamplitude EPSPs within the other investigated nuclei (PF, CL, and $\mathrm{AD}$ ), and we failed to detect unitary EPSPs in neurons from these nuclei either automatically or manually (Fig. 8A). 


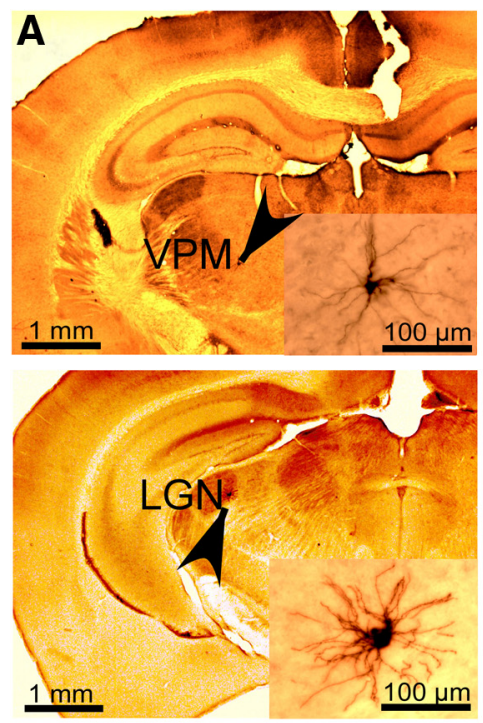

B

C
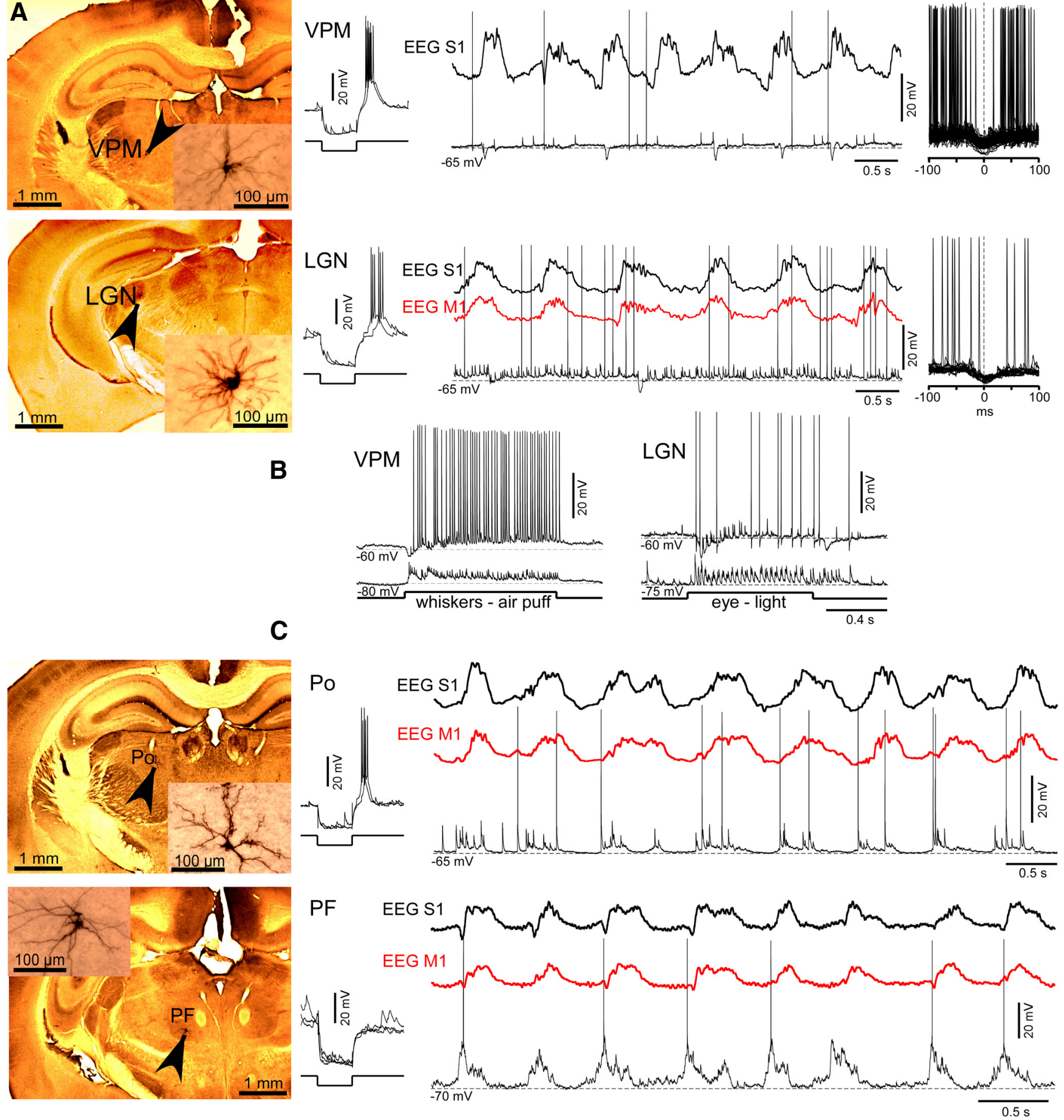

Figure 6. Two thalamic slow-wave patterns recorded from the first- and higher-order nuclei. $A$, Left, Light microscopic images of the intracellularly stained VPM and LGN neurons. There is a specific first-order "bushy" morphology of the neurons. The neurons revealed LTS in response to hyperpolarizing (100 ms, $1 \mathrm{nA})$ current pulses. Middle, Simultaneously recorded cortical contralateral EEG and intracellular activities from VPM and LGN nuclei. Both neurons displayed unitary IPSPs during cortical active states and "fast-rising" EPSPs, which were not modulated by cortical states. Right, Selected IPSPs. B, Contralateral sensory stimulation (whiskers and hair on the muzzle-air puff, eye-flash) elicited synaptic responses consisting of IPSPs and fast-rising EPSPs in VPM and LGN neurons. Top and bottom traces represent the responses of the neurons to sensory stimulation at depolarized and hyperpolarized membrane potential respectively with injection of steady inward and outward current of $0.5 \mathrm{nA}$. C, Left, Light microscopic images of the intracellularly stained PO and PF neurons. In contrast to PO neurons, PF neurons did not reveal LTS in response to hyperpolarizing (100 ms) current pulses. Right, Simultaneously recorded cortical EEG and intracellular activities from PO and PF nuclei. Both neurons displayed the slow-wave pattern with depolarization during active states.

To reveal slow-wave modulation of the thalamic and cortical synaptic activities, we plotted phase and time PSP (and/or synaptic noise) histograms (Fig. 9). We also analyzed spiking activities. All detected cortical and thalamic PSPs were modulated by slow waves, with the exception of large-amplitude EPSPs (drivers) re- corded within VPM and LGN nuclei (Fig. 9A). PSPs and spikes of all cortical cells as well as from all the neurons within thalamic $\mathrm{PO}$ ( $n=7$ neurons), PF $(n=6), \mathrm{AV}(n=7), \mathrm{CL}(n=1$, data not shown), and $\mathrm{AD}(n=1$, data not shown) nuclei were phaselocked ( $p<0.05$, Moore-Rayleigh test). Extracted IPSPs from 



Figure 7. Membrane potentials of thalamic and cortical cells are modulated by slow oscillation. Averaged membrane potentials of neurons recorded in the cortex (FrA-0, M1, S1, V1, V-RS, and V-RS posterior: $n=18,8,11,5,7$, and 7 neurons, respectively) and thalamus (PF, P0, AV, VPM, and LGN: $n=6,7,7,14$, and 6 neurons, respectively) plotted against phase. In all panels, the positive part of SEM is shown with gray (negative part is not shown). The cortical areas are schematically shown in the left.

$\operatorname{VPM}(n=14$ neurons) and LGN $(n=6)$ neurons were also phase-locked ( $p<0.05$, Moore-Rayleigh test). In 4 of 14 analyzed VPM neurons, we extracted phase-locked small-amplitude EPSPs/noise corresponding to modulators (Figs. $8 A$ and Fig. $9 A$, bottom, dotted black line, $p<0.05$, Moore-Rayleigh test). However, when all large-amplitude EPSPs (corresponding to thalamic drivers) or small- and large-amplitude EPSPs were extracted together, they were not phase-locked in the VPM (Fig. 9A, bottom, solid black line). We failed to extract small-amplitude EPSP/ noise modulated by cortical slow waves in LGN neurons, which were shown in Figure $8 A$. All EPSPs from that nucleus were not phase-locked to the slow oscillation (Fig. 9A, bottom, solid red line). Spike activities of only three VPM and no LGN neurons were phase-locked to the slow oscillation $(p<0.05$, MooreRayleigh test; data not shown).

\section{Global slow oscillation dynamics in the mouse thalamocortical system}

The averaged membrane potentials (Fig. 7) and PSP histograms (Fig. 9) revealed intacellular slow-wave dynamics in the thalamocortical system. The earliest structures involved in the generation of slow waves were the FrA-O and M1 cortices as well as PF and PO nuclei. Then, the intracellular active states propagated in the anteroposterior direction that was also shown with LFP recordings (Fig. 3E). The latest structures involved into the slow oscillation were V-RS posterior cortex and AV nucleus with an overall delay of approximately half of a cycle from the earliest structures. The mean phases (and significant differences between them) of membrane potentials, EPSPs, and spike histograms are shown in Tables 2 and 3. Both PF and PO half rising times were significantly different compared with rising times from S1, V1, V-RS, and V-RS posterior cortical neurons (Table 3 ). The mean PF phases obtained from EPSP histogram and from membrane potentials significantly differed from all the corresponding cortical mean phases $(p<0.05$, Watson-Williams test, Table 2$)$. The mean phases depend on the waveform and its duration. A more precise analysis representing the order of activation was obtained from the velocity of slow-wave activation (depolarization) plots (Fig. 10A, B; same data as in Figs. 7 and 9). The phase shifts were clearly revealed between thalamic and cortical neuron activation (Fig. 10A,B). The cortical mean phases of membrane potential derivatives $(>0)$ and EPSP histogram derivatives $(>0)$ were significantly different compared with each other respectively, except FrA-O versus M1 $(p<0.05$, Watson-Williams test; mean phases of individual neurons are shown in Fig. 10A2,B2). The thalamic $\mathrm{PF}$ mean phases of membrane potential derivatives $(>0)$ were significantly different from all the corresponding cortical mean phases ( $p<0.05$, Watson-Williams test). The thalamic PO mean phases of membrane potential derivatives $(>0)$ were not significantly different from FrA-O and M1 phases. The thalamic PF and PO mean phases of EPSP histogram derivatives $(>0)$ were significantly different from the corresponding mean S1, V1, V-RS, and $\mathrm{V}$-RS posterior phases ( $p<0.05$, Watson-Williams test) but were not significantly different from FrA-O and M1 phases. Thus, the phases obtained from the membrane potential derivatives revealed that PF and PO led the slow-wave cycle, whereas the phases from EPSP histograms showed a synchrony between thalamic PF/PO and the frontal cortical areas. In Figure 10E, the membrane potential (left) and EPSP histogram (right) derivatives $(>0)$ of PF and orbital cortex are plotted together, and their peaks are matched. The apparent statistical difference between PF and the frontal cortical areas obtained from the membrane potential derivatives is rather the result of the waveform and its duration since the peaks of activation occurred simultaneously (Fig. 10E). Taking into account the precision of the method (e.g., filtering, smoothing, Hilbert transformation), we conclude here that, on average, the thalamic PF and PO neurons started activation/depolarization simultaneously with the frontal cortical areas and before the other investigated parts of the cortex. Thalamic AV neurons were activated significantly after all other cortical and thalamic neurons, except V-RS posterior phase (Fig. 10A, B; Tables 2 and 3).

We also found a significant phase shift between IPSPs within VPM and LGN nuclei (VPM vs LGN mean phases: -0.326 vs $0.145, p<0.05$, Watson-Williams test; Fig. 9A). The IPSPs from the VPM nucleus occurred significantly later than M1 (but not S1) cortex activation (mean phases, $p<0.05$, Watson-Williams test) and IPSPs from the LGN nucleus occurred after the activation of both M1 and S1 (but not V1) cortices (mean phases, $p<$ 0.05 , Watson-Williams test). The inhibition within the sensory thalamus occurred statistically simultaneously with the activation of the corresponding primary cortical areas.

Despite an overt anteroposterior propagation of active state onsets, the termination of cortical active states occurred almost simultaneously in all investigated areas (Figs. 3E, 7, and 9). To support further this observation, we compared the velocities of active state termination in different cortical areas (negative components of the first derivatives; Fig. 10C,D). The averaged curves of cortical membrane potential and EPSP histogram derivatives $(<0)$ did not reveal clear shifts between their peaks and in right parts indicating tendency to terminate simultaneously (Fig. $10 C 1, D 1)$. Active state rising time or phase measured at half amplitude of histograms shown in Figure 9 revealed remarkable propagation, whereas decay time measured at half amplitude did not show significant propagation (Fig. 11A). Termination phase measured at half amplitude showed significant differences between frontal and posterior parts of the cortex, but the significance decreased when termination phase was measured at onetenth amplitude that implies that measured differences depended 
A
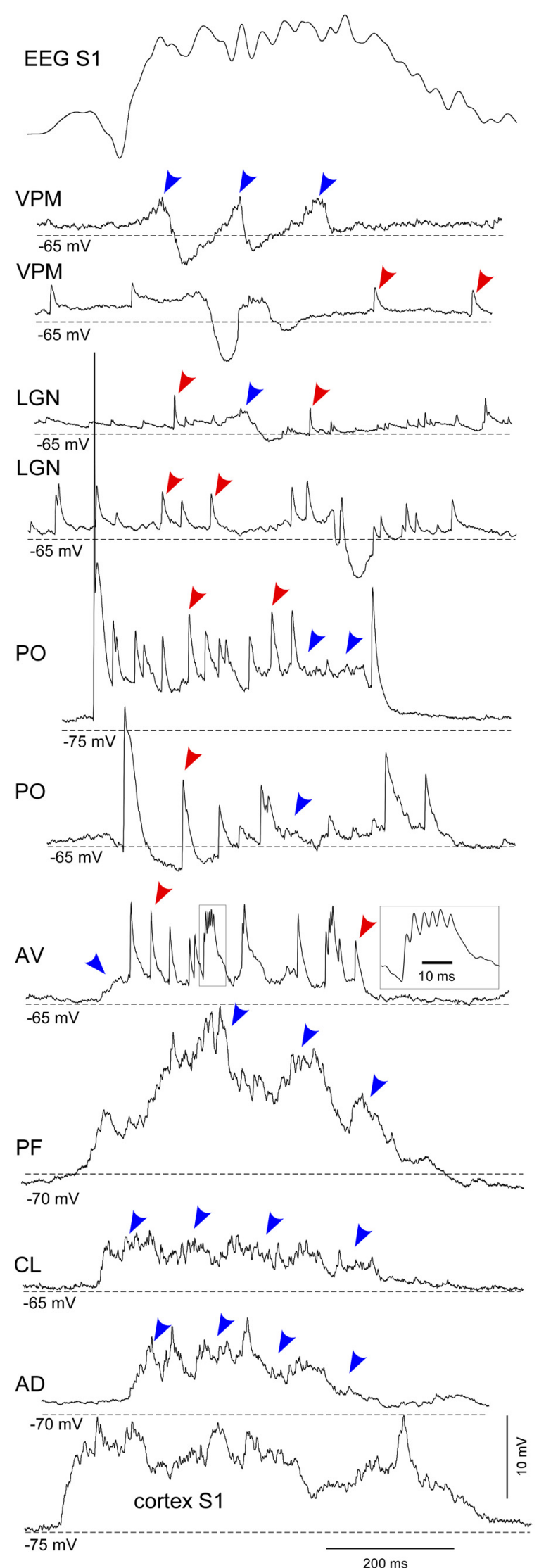

B

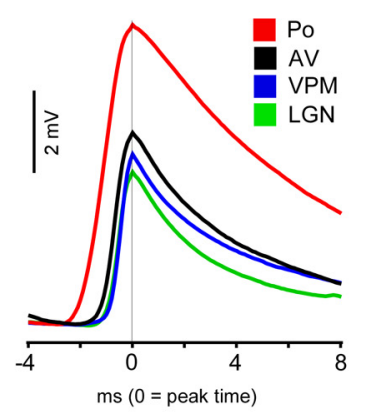

spontaneous evoked

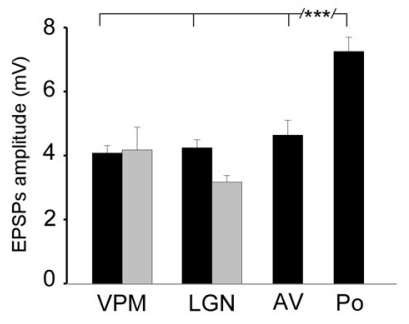

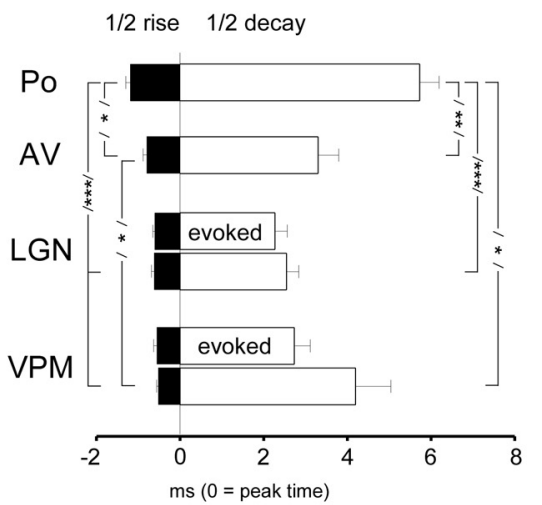

Figure 8. Drivers and modulators during slow oscillations in the mouse thalamus. A, Typical examples of neuronal activities recorded during slow-wave cycles from different thalamic nuclei and from the somatosensory cortex. The traces were aligned according to contralateral EEG (top). Red arrows and blue arrows indicate examples of unitary large-amplitude and merged together small EPSPs, respectively. Spontaneous large-amplitude EPSPs were a characteristic feature of all intracellular activities recorded within VPM, LGN, P0, and (Figure legend continues.) 
A

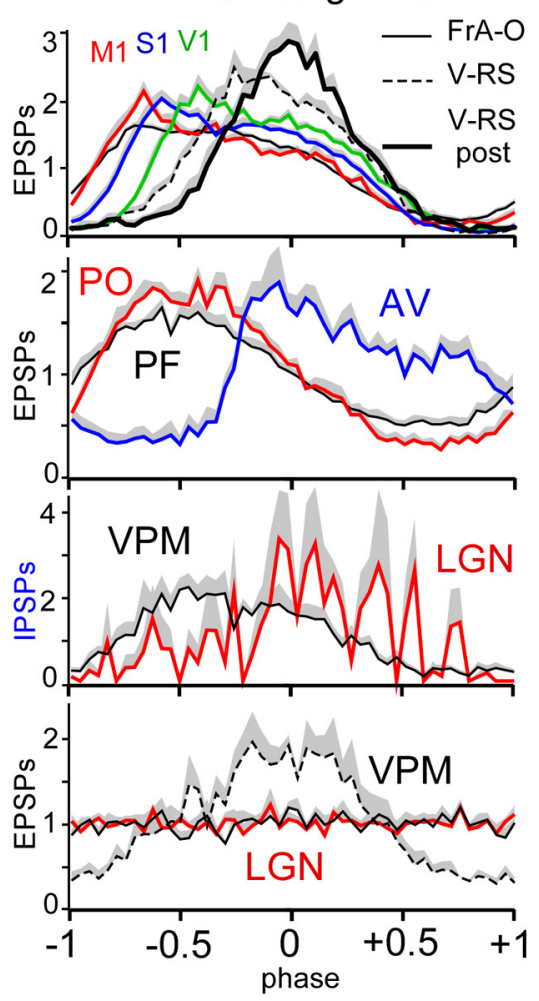

B
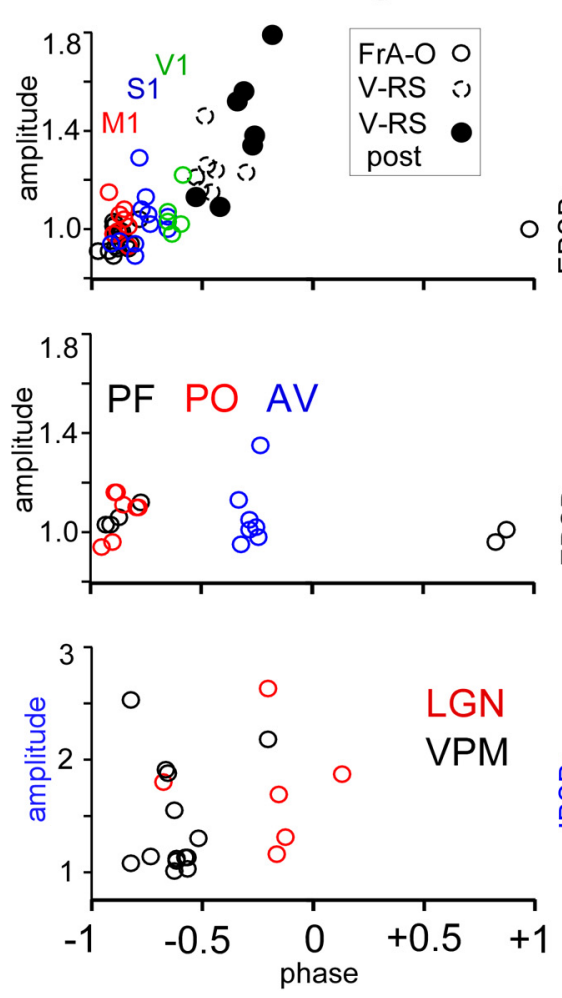

C

time PSPs histograms
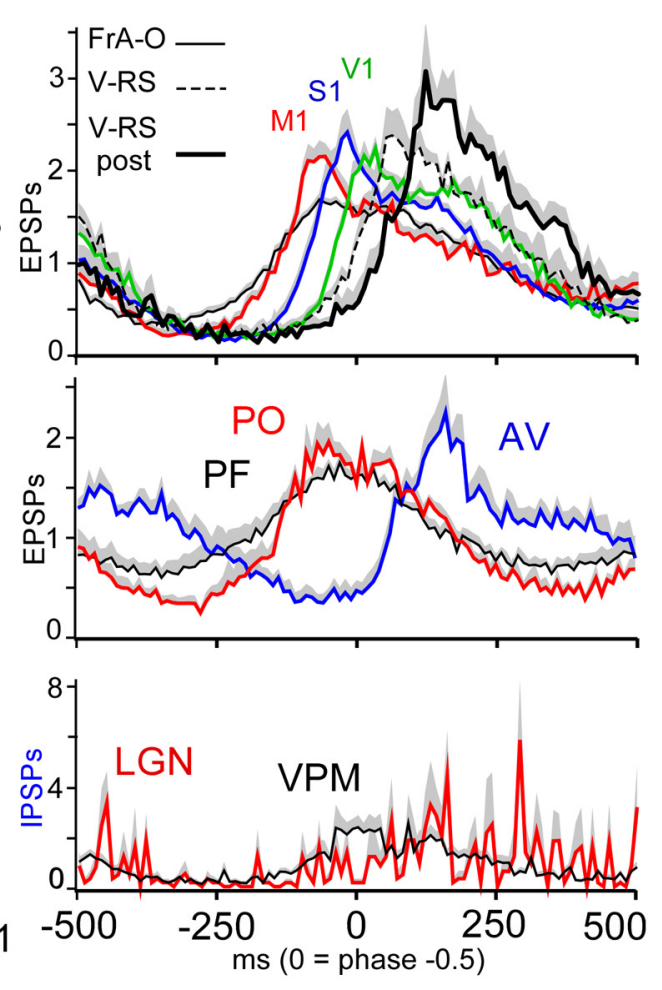

Figure 9. Synaptic activities during slow oscillations in the mouse thalamus and cortex. A, Averaged PSP histograms of the neurons recorded in the cortex (FrA-0, M1, S1, V1, V-RS, and V-RS posterior: $n=$ $18,8,11,5,7$, and 7 neurons, respectively) and thalamus (PF, P0, AV, VPM, and LGN: $n=6,7,7,14$, and 6 neurons, respectively) plotted against phase. Here and below before grand averaging, the histogram for each neuron was normalized by the mean value (each bin of the histogram was divided by the value of mean bin of the histogram). Bottom, The histograms for small-amplitude EPSPs extracted in 4 VPM neurons are shown with a dotted line. The histograms for fast-rising EPSPs extracted in VPM and LGN neurons are shown with a solid line. $\boldsymbol{B}$, Half rising amplitude of PSP histograms of each neuron is plotted against phase (averaged mean phases of the same points are shown in Fig. 11B). Bottom, Half rising amplitude of IPSP histograms of the VPM and LGN neurons is plotted against phase. C, Time PSP histograms. Zero time corresponds to the instantaneous phase -0.5 . In all panels, the positive part of SEM is shown with gray (negative part is not shown).

on waveform (Fig. 11B). Similar to Figure $3 E 3$ and the related analysis, comparison of segregate points of histograms revealed differences in termination (Fig. 11B), but taking into account the whole process (Fig. 10C1,D1), we conclude that the active states from different cortical areas tended to terminate simultaneously. Thus, during the propagation of the leading edge in the anteroposterior direction, active state duration became progressively shorter (Fig. 11A). The active state duration within the V-RS posterior versus the duration within frontal areas was almost twice shorter ( $p \leq 0.001$; Fig. $11 A)$.

To verify the results of phase analysis, we performed paired intracellular recordings within the thalamus and the cortex (Fig. 12). The neuron from the anterior thalamus started active states hundreds of milliseconds after the cortical S1 neuron (Fig. 12A), whereas the posterior thalamic neuron (presumably PO) was activated before the cortical S1 neuron recorded simultaneously (Fig. 12B). The results of time analysis obtained with dual intracellular recordings support the findings obtained with phase analysis of neuronal activation.

\section{$\leftarrow$}

(Figure legend continued.) AV nuclei. AV neurons generated bursts of EPSPs. $\boldsymbol{B}$, Averaged largeamplitude EPSPs recorded from four thalamic nuclei (VPM, LGN, PO, and AV: $n=9,7,11$, and 10 neurons, respectively). The plots represent the mean amplitudes (right) and kinetics (bottom) of the EPSPs. ${ }^{*} p<0.05$ ( $t$ test or Mann-Whitney sum rank test). ${ }^{* *} p<0.01$ ( $t$ test or Mann-Whitney sum rank test). ${ }^{* * *} p<0.001$ ( $t$ test or Mann-Whitney sum rank test). Largeamplitude EPSPs within VPM and LGN neurons, which were not modulated by cortical states and responded to sensory stimulation displayed the shortest rising time (fast-rising EPSPs).
Comparison of the inhibitory inputs into projecting VPM and second-order PO nuclei

One of unexpected findings of the study was absence of welldefined inhibitory activities in most second-order thalamic nuclei. It is well documented that the main inhibitory input into first-order thalamic nuclei is provided by the RTN, whereas higher-order thalamus is also innervated with other subcortical nuclei (Barthó et al., 2002; Bokor et al., 2005). Particularly, the PO nucleus receives inhibitory input from the ZI (Trageser and Keller, 2004). As expected, in sensory thalamic nuclei, we found IPSPs phase-locked to the slow oscillation, but we did not find profound IPSPs within higher-order thalamic nuclei during slow oscillations. Therefore, using retrograde labeling by $\mathrm{CtB}$, we investigated differences in the inputs from RTN and ZI into VPM and PO nuclei, respectively. CtB injected in the VPM nucleus ( $n=7$ mice) retrogradely labeled neurons within the cortical layer VI of the somatosensory cortex as well as neurons in the RTN (Fig. 13A), whereas, when injected into PO ( $n=6$ mice), $\mathrm{CtB}$ labeled neurons mainly within the cortical layer $\mathrm{V}$ and in the ZI (Fig. 13B). The labeled cells within the RTN tended to be grouped in clusters, whereas labeled neurons within the ZI tended to stain as individual neurons (Fig. 13). We used an unbiased automatic quantification of all labeled objects (individual neurons or clusters of neurons, area of $>40 \mu \mathrm{m}^{2}$ ) from the sections containing the RTN or the ZI (Fig. 13C). To quantify differences between local densities of stained neurons within VPM and ZI nuclei, we calculated the ratio between the integrated areas 


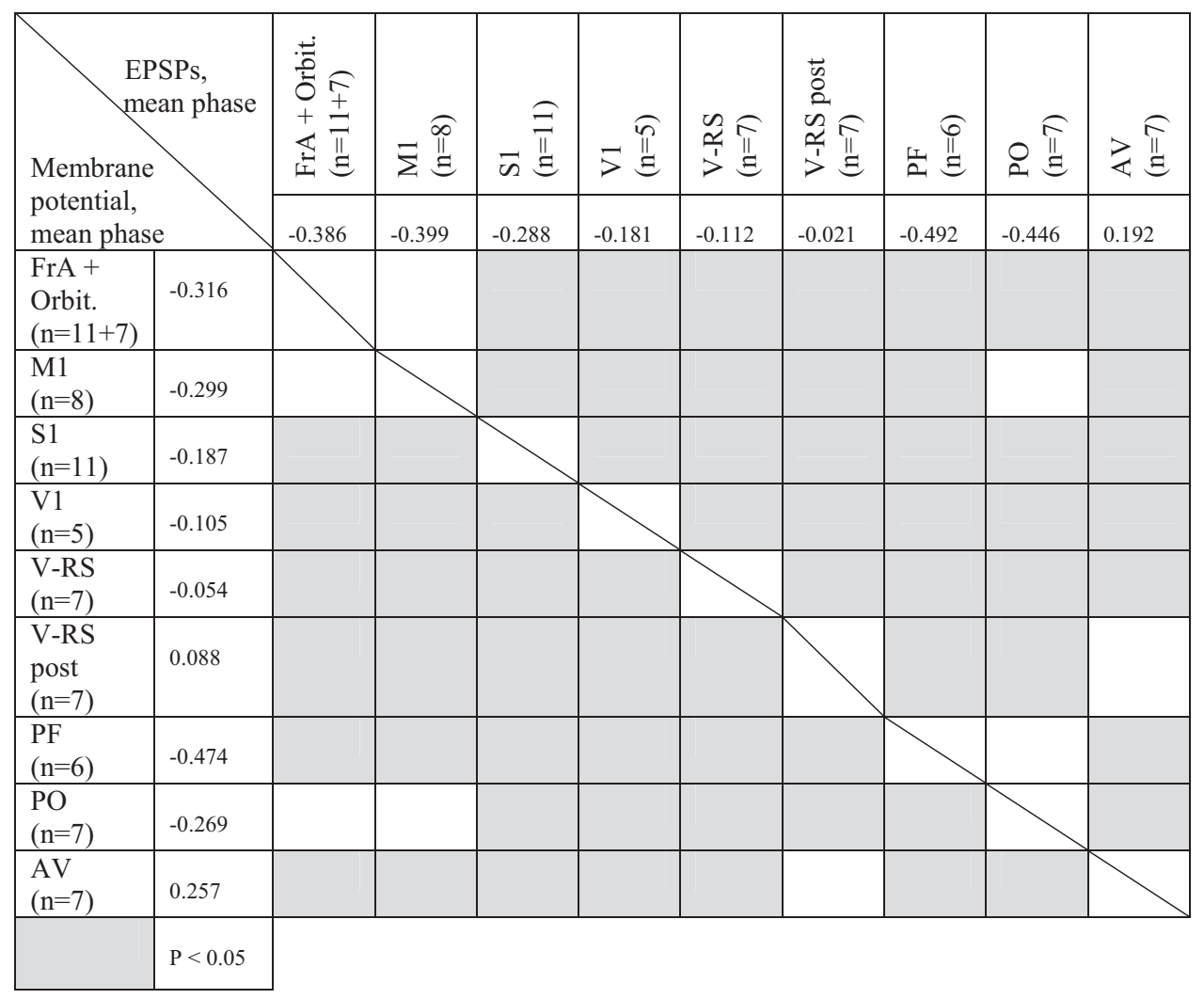

Table 2. The number of neurons are indicated. Above the diagonal: significant differences between the mean phases from EPSP histograms (Fig. $9 A$ ) are shown by gray. Below the diagonal: significant differences between the mean phases from the averaged membrane potentials (Fig. 7 ) are shown by gray. $p<0.05$ (Watson-Williams test).

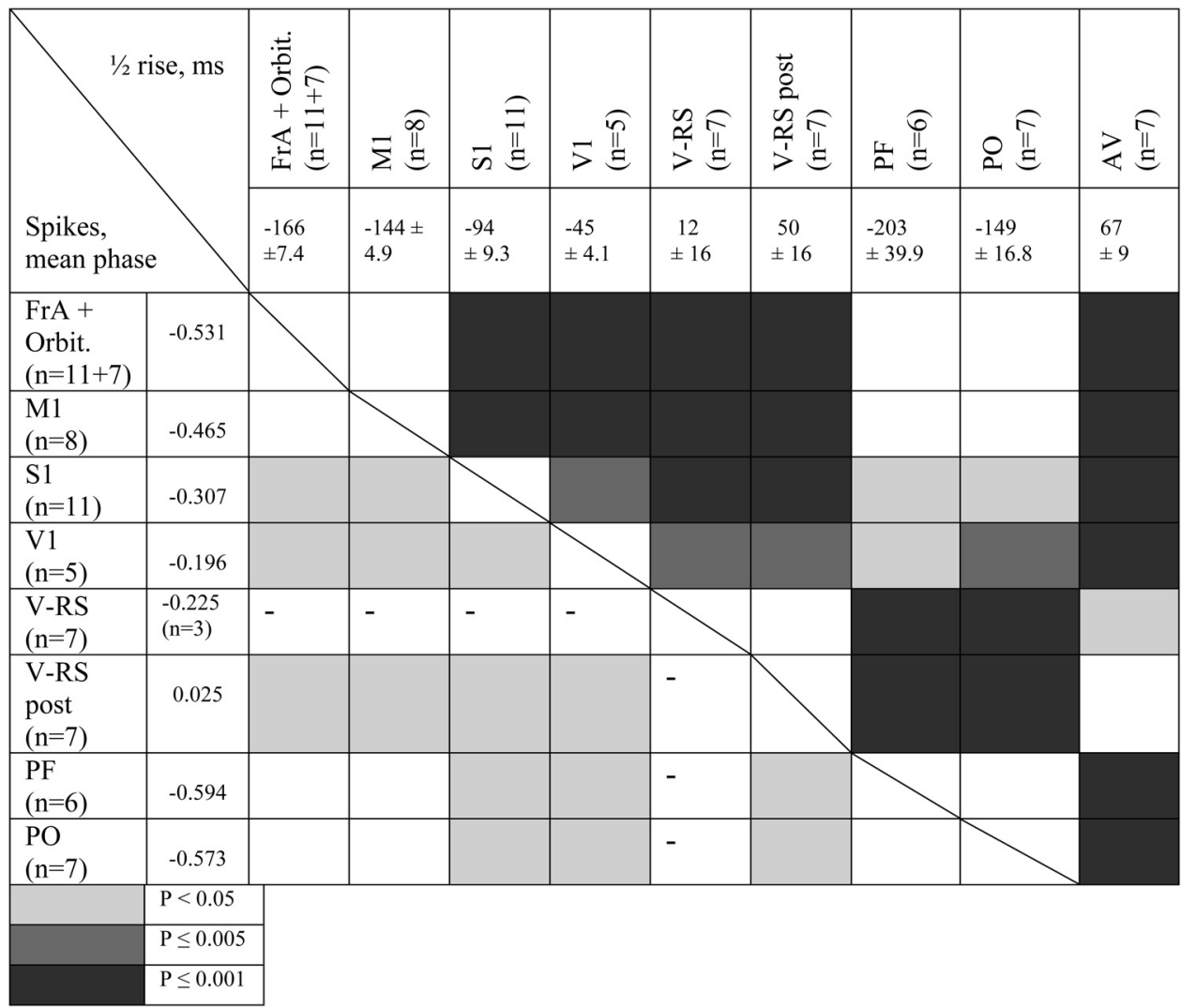

Table 3. The numbers of neurons are indicated. Above the diagonal: significant differences between the mean $1 / 2$ rising times obtained from time histograms (represented as mean \pm SEM, Figs. $9 B$ and $11 A$ ). Below the diagonal: significant differences between the mean phases obtained from spike histograms (not shown). Differences are shown with different intensities of gray (for the phases, Watson-Williams test; for the times, two-tailed $t$ test or Mann-Whitney test). 
A1 B1

C1

D1
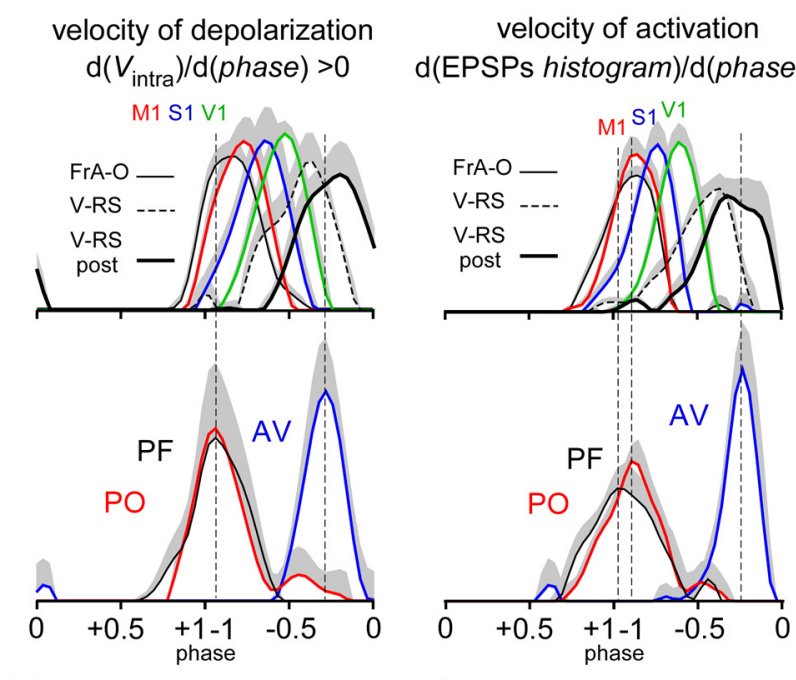

velocity of hyperpolarization

velocity of termination $\mathrm{d}\left(V_{\text {intra }}\right) / \mathrm{d}($ phase $)<0$

$\mathrm{d}($ EPSPs histogram $) / \mathrm{d}($ phase $)<0$

\section{A2}

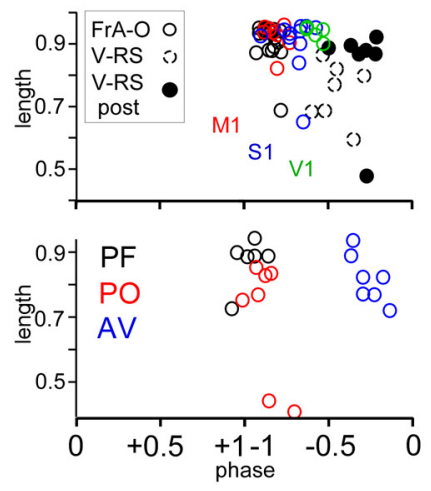

\section{B2}
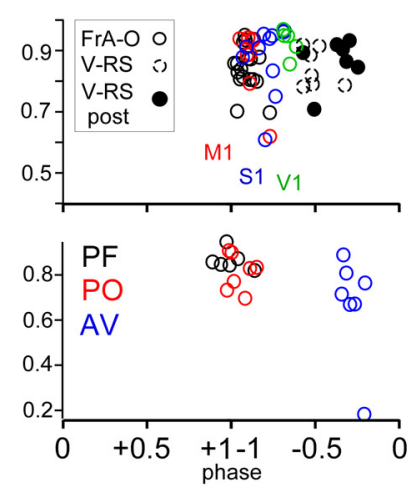
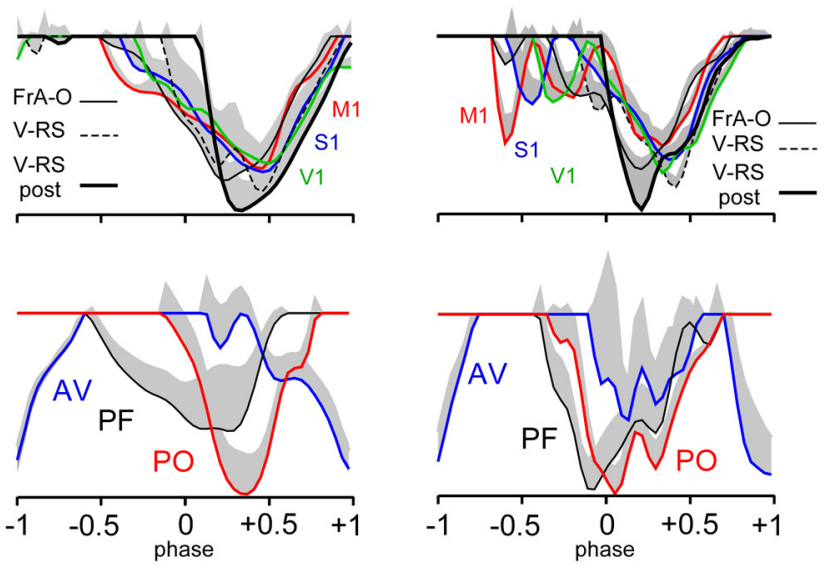

\section{C2}
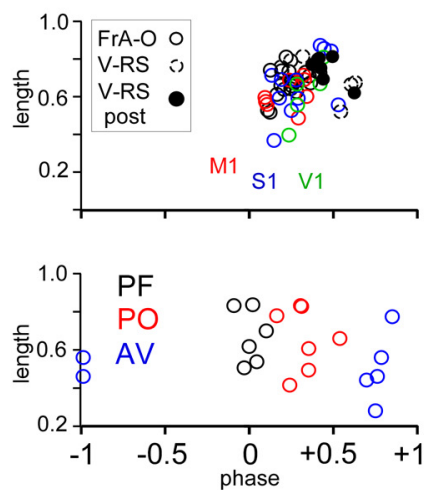

D2
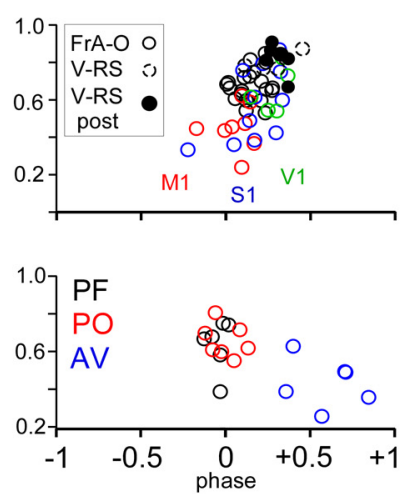

\section{E}
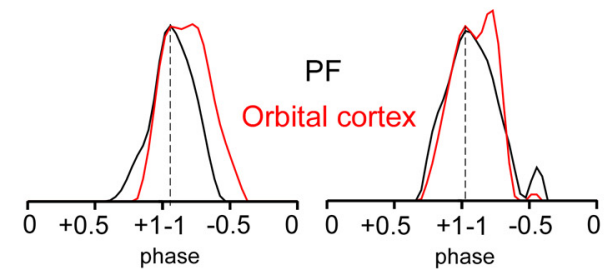

F

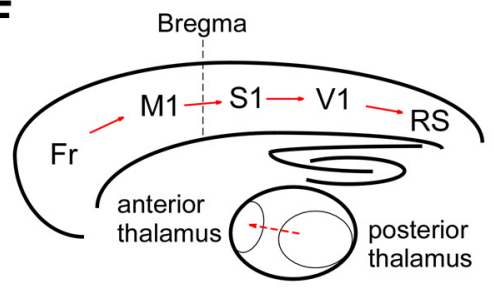

Figure 10. Global slow-wave dynamics within the thalamocortical system of a mouse. A1, Differentiated by phase membrane potentials are plotted against phase of EEG slow oscillation. The averaged positive component of the derivative is shown, which represents the velocity of depolarization in cortical and thalamic neurons. Here and below before grand averaging, the plot for each neuron was normalized by the mean value. $\mathbf{A}$, The mean vectors (length) of the same components as in $\boldsymbol{A} \mathbf{1}$ are shown for individual neurons, which represent the mean phase of depolarization of the neurons during slow wave. $\boldsymbol{B}$ 1, Differentiated by phase, phase EPSP histograms are plotted. The averaged positive component of the derivative represents the increments of EPSP number during transitions to the active states. $\boldsymbol{B 2}$, The mean vectors (length) of the same components as in $\boldsymbol{B} 1$ are shown for individual neurons, which represent the mean phase of the increments of EPSP number during transitions to the active states. C1, The averaged negative component of the derivative represents the velocity of hyperpolarization in cortical and thalamic neurons. C2, The mean vectors (length) of the same components as in $\mathbf{C}$ are shown for individual neurons, which represent the mean phase of hyperpolarization during the transition from active to silent state. D1, The velocity of termination of active states estimated from EPSPs histograms for cortical neurons (top) and thalamic neurons (bottom). D2, The mean vectors (length) of the same components as in D1 are shown for individual neurons, which represent the mean phase of EPSPs active state termination. $\boldsymbol{E}$, The positive components of derivatives from the thalamic PF and orbital cortical neurons are plotted together, and their amplitudes are normalized by maximum of the first peak (left, obtained from membrane potentials; right, from EPSP histograms). In all panels, the positive part of SEM is shown with gray (negative part is not shown). $\boldsymbol{F}$, The sequence of detection of slow waves in the cortex and thalamus is schematically shown.

of clusters (object length $>25 \mu \mathrm{m}$ ) to the total area of all labeled objects for each animal. The ratios for RTN and ZI nuclei were $0.74 \pm 0.15$ versus $0.20 \pm 0.09$, respectively $(p \leq 0.001)$, reflecting the staining in clusters of neurons within the RTN versus the staining of individual neurons in the ZI.

\section{Discussion}

In the present study, we show that, in the mouse thalamus, during active phases of the slow oscillation, the inhibition dominates in sensory nuclei, whereas the excitation dominates in higher-order nuclei. Applying phase and time analysis to intracellular and LFP 
A
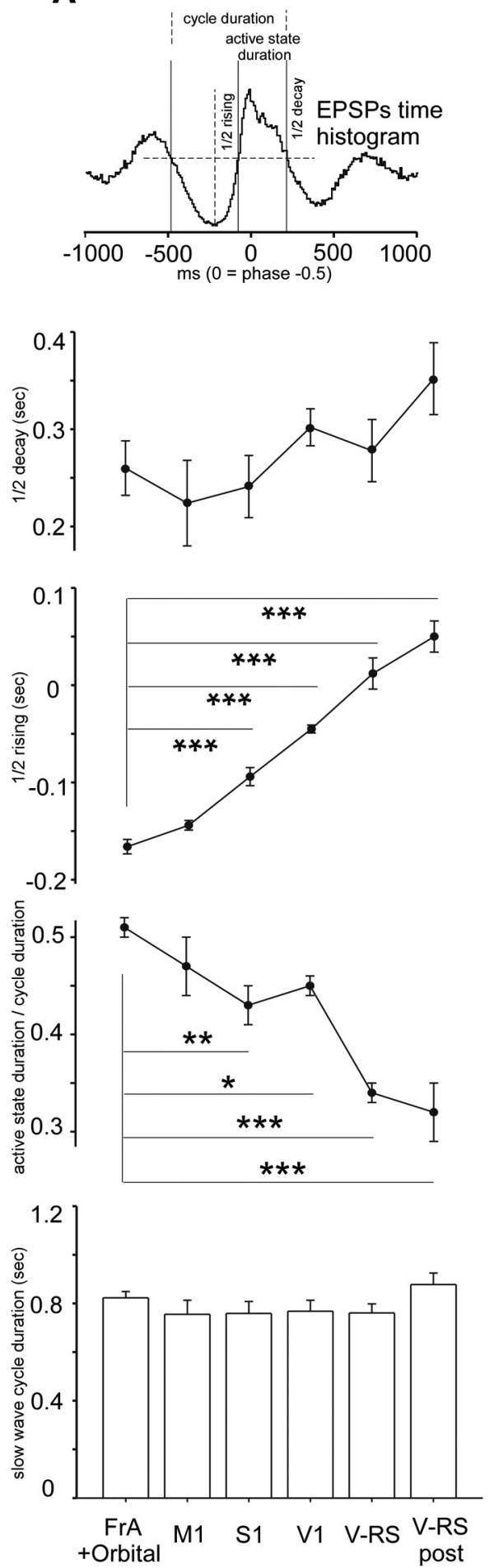

B
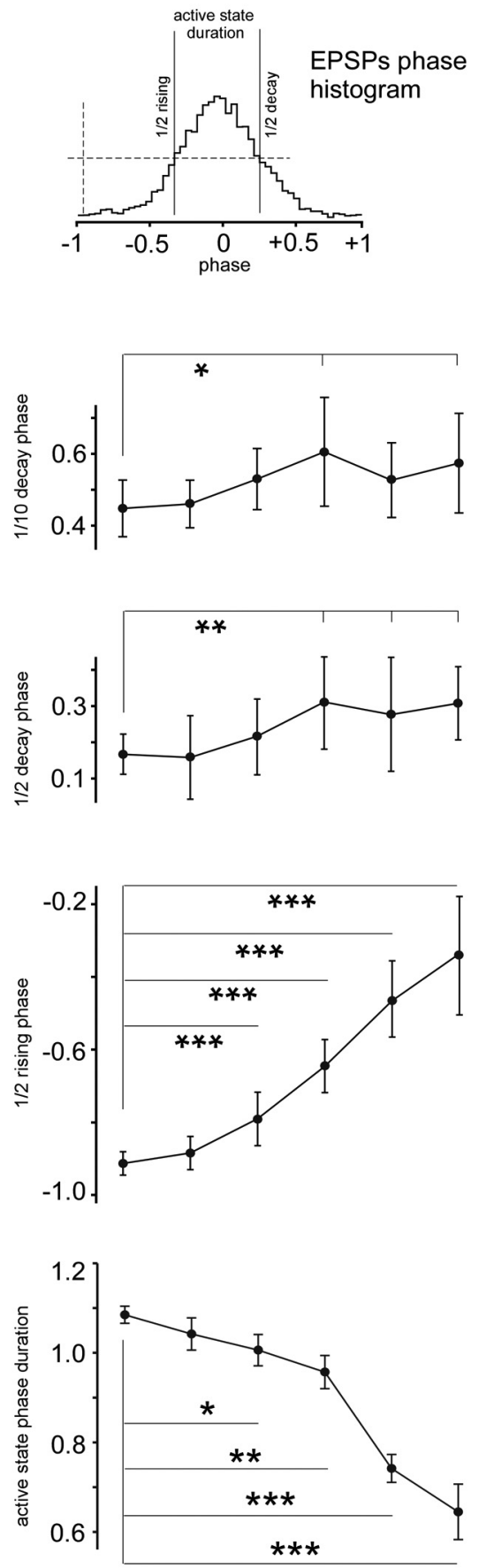

$\underset{+ \text { Orbital }}{\text { FrA }} \begin{array}{crrr}\text { S1 } & \text { V1 V-RS } \\ \text { p-RS } & \text { post }\end{array}$

Figure 11. Duration $(\boldsymbol{A})$ and proportion of phase $(\boldsymbol{B})$ of active states within different cortical areas. $\boldsymbol{A}$, Calculated parameters are shown schematically on top. Zero time was set at -0.5 of phase (Fig. 1). Active state duration was calculated as the time interval between half rising and half decay within EPSP time histograms. The slow-wave cycle duration was calculated as time between two consecutive crossings for decay. Active states become significantly shorter while propagating in the anteroposterior direction independently on the duration of slow-wave cycle. $\boldsymbol{B}$, Calculated parameters are shown schematically on top. One-tenth decay phase was measured similar to half decay. Proportion of phase occupied by active states was calculated from the interval between the half decay and half rising phases within EPSP phase histograms using basic statistics as in $A .{ }^{*} p<0.05$ ( $t$ test or Mann-Whitney sum rank test). ${ }^{* *} p<0.01$ ( $t$ test or Mann-Whitney sum rank test). ${ }^{* *} p \leq 0.001$ ( $t$ test or Mann-Whitney sum rank test). Half, one-tenth decay, and half rising phases were compared using circular statistics. ${ }^{*} p<0.05$ (Watson-Williams test). ${ }^{* *} p<0.01$ (Watson-Williams test). ${ }^{* * *} p \leq 0.001$ (Watson-Williams test). Data are mean \pm SEM (phase duration and time) and mean \pm confidence interval stated at the $95 \%$ confidence level (half decay and half rising phases).

activities within different cortical and thalamic areas, this study is the first to provide information on the global structure of the slow oscillation within the TC system of anesthetized mice. (1) Similar to previous studies (Massimini et al., 2004; Mohajerani et al., 2010; Nir et al., 2011; Stroh et al., 2013), we confirm that most of the slow oscillation cycles started from the frontal cortical areas and the leading edge of the slow waves propagated over the cortex in the anteroposterior direction, whereas the end of active states occurred nearly simultaneously in different cortical sites (Volgushev et al., 2006) (Figs. 3, 7, 9, and 10); (2) the first-order (sensory) thalamic nuclei followed the corresponding cortical areas during the slow oscillation; (3) the higher-order PO and intralaminar PF thalamic neurons started off the slow-wave active states approximately simultaneously; and (4) the latest structure to become involved in the slow-wave cycle was the anterior thalamus, which approximately followed the retrosplenial cortex.

Neurons within the sensory thalamus revealed IPSPs modulated by cortical slow waves as well as large-amplitude, fastrising EPSPs originating likely from ascending structures and therefore not modulated by the slow oscillation. When "driver" inputs arrived to TC neurons during IPSP, we never observed spikes triggered by the driver, which is consistent with the gating role of the slow oscillation (Timofeev et al., 1996). The inhibitory potentials in VPM and LGN neurons during the slow oscillation originated likely because of burst firing of RTN neurons (Contreras and Steriade, 1995; Timofeev and Steriade, 1996). Instead, the nonsensory thalamus revealed depolarizing active states and therefore was actively involved in the cortical slow oscillation by providing a phase-locked positive feedback and likely contributing to the synchronization of cortical active states.

The two intracellular slow-wave patterns we detected in the sensory versus higher-order thalamus of anesthetized mice could be explained by the patterns of their connectivity. (1) The drivers within sensory nuclei originate in ascending sensory pathways, whereas the drivers in higher-order nuclei originate from layer $\mathrm{V}$ pyramidal cells (Groh et al., 2008), which by themselves are the neurons playing a leading role in the generation of slow oscillation (Chauvette et al., 2010; Beltramo et al., 2013). (2) Consistent with the known ratio of large versus small excitatory synapses in different thalamic nuclei 
A
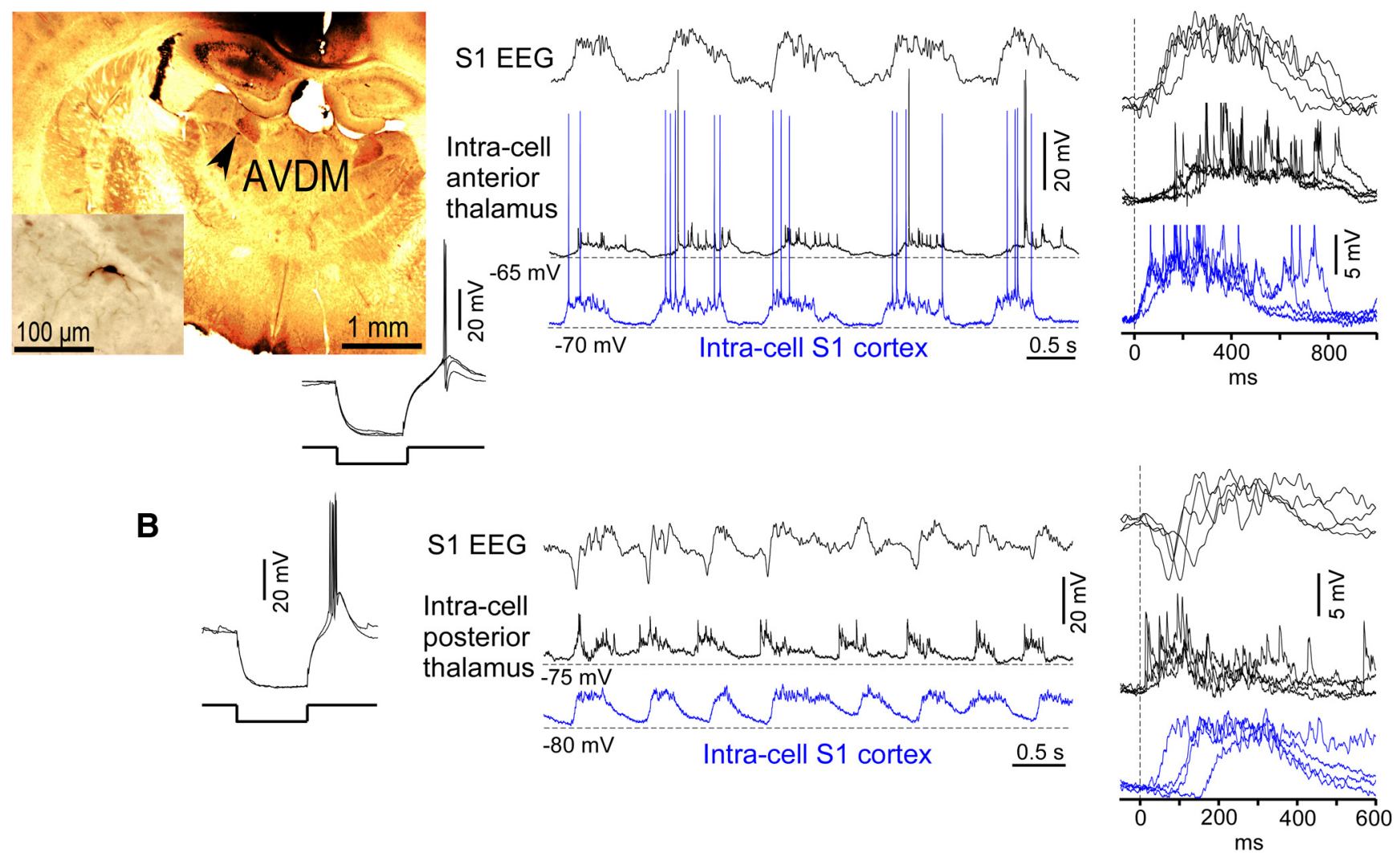

Figure 12. The two examples of paired intracellular recordings revealed different time delays between cortical $S 1$ cells and neurons from the anterior and posterior thalamus. $A$, The thalamic neuron was recorded within the dorsomedial part of anteroventral nucleus (AVDM). The thalamic active states started almost $200 \mathrm{~ms}$ after the cortical active states. $\boldsymbol{B}$, The thalamic active states started $\sim 100$ ms before the cortical active states. The thalamic neuron was not identified morphologically, but we targeted the posterior thalamus.
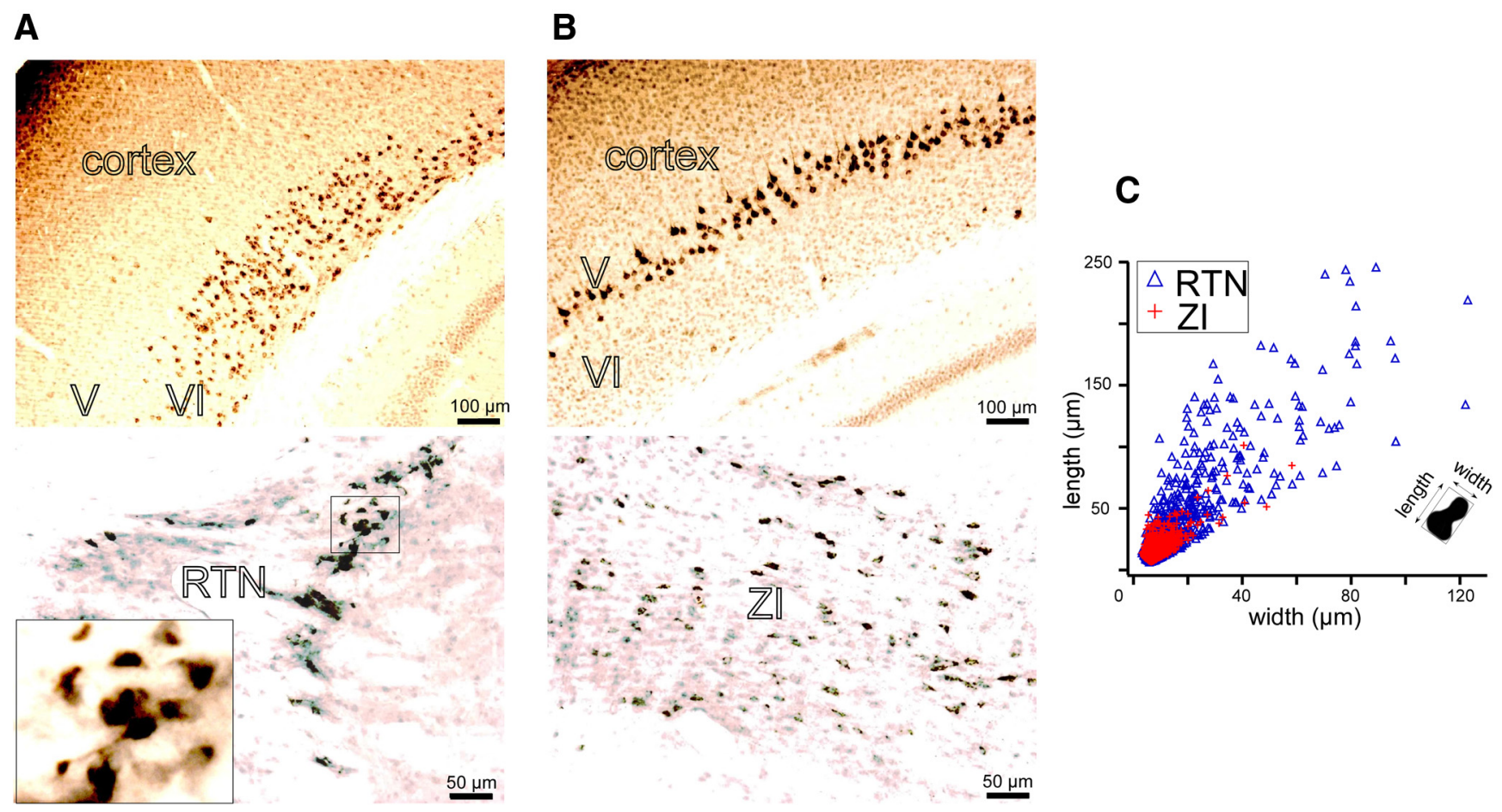

Figure 13. Comparison of the main inhibitory inputs projecting into first-order VPM and second-order P0 nuclei. $A$, CtB injected in the VPM retrogradely labeled neurons within the cortical layer VI as well as cells in the RTN. B, When CtB was injected into the PO nucleus, it labeled neurons within the cortical layer V and in ZI. Unlike Zl neurons, RTN-labeled cells tend to group together. C, Quantification of labeled object size within Zl and RTN nuclei. Labeled RTN neurons tend to form clusters. 
(Van Horn and Sherman, 2007), the activities of modulators were much more pronounced within higher-order nuclei than within sensory VPM and LGN nuclei. (3) It appears that the RTN provides a stronger inhibition into sensory thalamic nuclei during cortical active states than the ZI onto higher-order nuclei because a majority of RTN neurons fire bursts of action potentials (Avanzini et al., 1989; Contreras et al., 1992; Fuentealba et al., 2005), but ZI neurons fire tonically (Trageser et al., 2006). Therefore, the postsynaptic action of RTN neurons should be stronger compared with ZI neurons. Also, the RTN neurons form clusters (Fig. 13). It is known that RTN neurons are interconnected by chemical and electrical synapses (Landisman et al., 2002; Shu and McCormick, 2002). Clusters of electrically coupled RTN neurons generate synchronous activities (Long et al., 2004), suggesting that tightly interconnected bursting RTN neurons within clusters would induce stronger IPSPs in their targets than dispersed tonically firing neurons from ZI.

Consistent with morphological observations in primates (Rovó et al., 2012), we did not observe "driver" EPSPs within intralaminar PF neurons, but we observed it in the PO nucleus. The thalamic PO drivers provided a strong relay between the primary and the secondary somatosensory cortices (Theyel et al., 2010), contributing to the long-range cortical synchronization. The "driver" EPSPs within thalamic AV neurons probably originated from the hippocampal system and/or retrosplenial cortex (Shibata, 1998; Petrof and Sherman, 2009).

The neurons within PF, CL, and AD nucleus did not display LTS in response to a hyperpolarizing current pulse, corroborating previous extracellular unit and whole-cell in vitro studies (Lacey et al., 2007; Beatty et al., 2009). Interestingly, the same thalamic neurons in our study did not display driver EPSPs, suggesting their very particular role in the thalamic functions.

One of the intriguing findings of the study was that, on average, the onset of active states in PO and in PF neurons occurred simultaneously with the onset of earliest cortical active states. The onset of depolarizing states appeared as a buildup of synaptic "modulator" events in the PF nucleus or as large-amplitude EPSPs in the PO nucleus (Fig. 8), suggesting its origin in presynaptic structures. Cortical inputs to PF nucleus of rat arrive from primary and secondary motor cortex, primary somatosensory cortex, and parietal cortex (Cornwall and Phillipson, 1988; Van der Werf et al., 2002). The frontal cortical areas as well as the thalamus, forming long-distance and multiple connections, are considered to be hubs within the functional brain network (Honey et al., 2007; van den Heuvel and Sporns, 2011). If one assumes a probabilistic mechanism of slow-wave initiation at any cortical site (Timofeev et al., 2000; Chauvette et al., 2010), and the PF nucleus represents a hub connecting these cortical areas, which integrates multiple inputs from long-distance sites, the PF nucleus would be at least the second to activate during each slow-wave cycle and thus, in relation to all cycles together (average), it would be detected as the first structure. The PF nucleus also receives inputs from laterodorsal and pedunculopontine tegmentum, locus ceruleus, and raphe nuclei, most densely the dorsal raphe (Cornwall and Phillipson, 1988; Van der Werf et al., 2002). During slow-wave sleep, locus ceruleus neurons tend to fire before the onset of active states in the prefrontal cortex (Eschenko et al., 2012); therefore, they can actively contribute to the early onset of active states in PF neurons. Phase-locked firing of other neuromodulatory structures may also contribute to shaping of the slow oscillation.

Despite the fact that the PF neurons demonstrated the early onset of slow-wave active states, their role in the global slow-wave generation is unclear. The majority of projections from the PF nucleus reach striatum (Deschênes et al., 1996; Van der Werf et al., 2002). The cortical projections of these neurons form small terminal puffs in layer VI of the frontal motor cortex (Deschênes et al., 1996). These data indicate that PF neurons can boost the slow oscillation mainly in frontal areas.

Neurons of the PO nucleus receive ascending inputs from somatosensory, auditory, visual, and vestibular systems (Groenewegen and Witter, 2004), which should not be synchronized with the slow oscillation. The cortical (driver) inputs arrive mainly from somatosensory (S1 and S2) areas, but to a lesser extent from medial frontal cortices (Veinante et al., 2000; Guandalini, 2001); and frontal cortex is the first to display the onset of slow waves, which potentially could drive these neurons and show the early onset of activity in the PO nucleus. However, the density of corticothalamic terminals originating from the frontal cortex to PO and AV nuclei is similar (Guandalini, 2001); the PO nucleus was among the first to enter into active phases of the slow oscillation and the AV nucleus was the last one (Fig. 10).

This and other studies (Massimini et al., 2004; Volgushev et al., 2006; Ruiz-Mejias et al., 2011) show that cortical active state onsets propagate in the anteroposterior direction as well as demonstrate simultaneous termination of active states across neocortex. Although the exact mechanisms of this simultaneous termination remain unknown, several investigations point to a possible role of inhibitory processes in such termination (Steriade et al., 1993; Puig et al., 2008; Chen et al., 2012). Active contribution of higher-order thalamocortical neurons in active state maintenance suggests that some of them can provide a small, but widespread, influence over distant cortical areas and therefore control termination of active states.

In this study using stereotypic global slow-wave pattern in ketamine-xylazine anesthetized mice, we show that the active phases of slow waves occur first in frontal cortical areas and in second-order (posterior) thalamic nuclei (PF and PO). The slow waves propagate in the rostrocaudal direction in the cortex (from frontal to retrosplenial cortex). The sequence of detection of slow waves in the thalamus was in the caudorostral direction (from posterior PF and PO nuclei to the anterior AV nucleus; Fig. 10F). The AV nucleus forms reciprocal connections with the limbic system and, in particular, with the retrosplenial cortex (Groenewegen and Witter, 2004) as well as the medial prefrontal cortex (Guandalini, 2001), potentially making a link between cortical areas with the earliest and the latest involvement in the slow oscillation. Our results demonstrate that only the first-order nuclei might contribute to the sleep-related gating of peripheral inputs, whereas higher-order thalamic nuclei may facilitate an intracortical dialog during sleep, which is possibly a key component of sleep-dependent memory consolidation (Rasch and Born, 2013).

\section{References}

Avanzini G, de Curtis M, Panzica F, Spreafico R (1989) Intrinsic properties of nucleus reticularis thalami neurones of the rat studied in vitro. J Physiol 416:111-122. Medline

Barthó P, Freund TF, Acsády L (2002) Selective gabaergic innervation of thalamic nuclei from zona incerta. Eur J Neurosci 16:999-1014. CrossRef Medline

Beatty JA, Sylwestrak EL, Cox CL (2009) Two distinct populations of projection neurons in the rat lateral parafascicular thalamic nucleus and their cholinergic responsiveness. Neuroscience 162:155-173. CrossRef Medline

Beltramo R, D’Urso G, Dal Maschio M, Farisello P, Bovetti S, Clovis Y, Lassi G, Tucci V, De Pietri Tonelli D, Fellin T (2013) Layer-specific excitatory circuits differentially control recurrent network dynamics in the neocortex. Nat Neurosci 16:227-234. CrossRef Medline 
Bokor H, Frère SG, Eyre MD, Slézia A, Ulbert I, Lüthi A, Acsády L (2005) Selective GABAergic control of higher-order thalamic relays. Neuron 45: 929-940. CrossRef Medline

Bourassa J, Pinault D, Deschênes M (1995) Corticothalamic projections from the cortical barrel field to the somatosensory thalamus in rats: a single-fibre study using biocytin as an anterograde tracer. Eur J Neurosci 7:19-30. Medline

Chauvette S, Volgushev M, Timofeev I (2010) Origin of active states in local neocortical networks during slow sleep oscillation. Cereb Cortex 20: 2660-2674. CrossRef Medline

Chauvette S, Crochet S, Volgushev M, Timofeev I (2011) Properties of slow oscillation during slow-wave sleep and anesthesia in cats. J Neurosci 31: 14998-15008. CrossRef Medline

Chen JY, Chauvette S, Skorheim S, Timofeev I, Bazhenov M (2012) Interneuron-mediated inhibition synchronizes neuronal activity during slow oscillation. J Physiol 590:3987-4010. CrossRef Medline

Constantinople CM, Bruno RM (2013) Deep cortical layers are activated directly by thalamus. Science 340:1591-1594. CrossRef Medline

Contreras D, Steriade M (1995) Cellular basis of EEG slow rhythms: a study of dynamic corticothalamic relationships. J Neurosci 15:604-622. Medline

Contreras D, Curró Dossi R, Steriade M (1992) Bursting and tonic discharges in two classes of reticular thalamic neurons. J Neurophysiol 68: 973-977. Medline

Contreras D, Timofeev I, Steriade M (1996) Mechanisms of long-lasting hyperpolarizations underlying slow sleep oscillations in cat corticothalamic networks. J Physiol 494:251-264. Medline

Cornwall J, Phillipson OT (1988) Afferent projections to the parafascicular thalamic nucleus of the rat, as shown by the retrograde transport of wheat germ agglutinin. Brain Res Bull 20:139-150. CrossRef Medline

Cossart R, Aronov D, Yuste R (2003) Attractor dynamics of network up states in the neocortex. Nature 423:283-288. CrossRef Medline

Crunelli V, Hughes SW (2010) The slow $(<1 \mathrm{~Hz})$ rhythm of non-REM sleep: a dialogue between three cardinal oscillators. Nat Neurosci 13:917. CrossRef Medline

David F, Schmiedt JT, Taylor HL, Orban G, Di Giovanni G, Uebele VN, Renger JJ, Lambert RC, Leresche N, Crunelli V (2013) Essential thalamic contribution to slow waves of natural sleep. J Neurosci 33:1959919610. CrossRef Medline

Deschênes M, Bourassa J, Pinault D (1994) Corticothalamic projections from layer $\mathrm{V}$ cells in rat are collaterals of long-range corticofugal axons. Brain Res 664:215-219. Medline

Deschênes M, Bourassa J, Doan VD, Parent A (1996) A single-cell study of the axonal projections arising from the posterior intralaminar thalamic nuclei in the rat. Eur J Neurosci 8:329-343. CrossRef Medline

Eschenko O, Magri C, Panzeri S, Sara SJ (2012) Noradrenergic neurons of the locus coeruleus are phase locked to cortical up-down states during sleep. Cereb Cortex 22:426-435. CrossRef Medline

Fuentealba P, Timofeev I, Bazhenov M, Sejnowski TJ, Steriade M (2005) Membrane bistability in thalamic reticular neurons during spindle oscillations. J Neurophysiol 93:294-304. Medline

Groenewegen HJ, Witter MP (2004) Thalamus. In: The rat nervous system, Ed 3 (Paxinos G, ed.), pp 407-453. New York: Elsevier Academic.

Groh A, de Kock CP, Wimmer VC, Sakmann B, Kuner T (2008) Driver or coincidence detector: modal switch of a corticothalamic giant synapse controlled by spontaneous activity and short-term depression. J Neurosci 28:9652-9663. Medline

Guandalini P (2001) The efferent connections to the thalamus and brainstem of the physiologically defined eye field in the rat medial frontal cortex. Brain Res Bull 54:175-186. CrossRef Medline

Honey CJ, Kötter R, Breakspear M, Sporns O (2007) Network structure of cerebral cortex shapes functional connectivity on multiple time scales. Proc Natl Acad Sci U S A 104:10240-10245. CrossRef Medline

Hughes SW, Cope DW, Blethyn KL, Crunelli V (2002) Cellular mechanisms of the slow $(<1 \mathrm{~Hz})$ oscillation in thalamocortical neurons in vitro. Neuron 33:947-958. CrossRef Medline

Jones EG (2009) Synchrony in the interconnected circuitry of the thalamus and cerebral cortex. Ann N Y Acad Sci 1157:10-23. CrossRef Medline

Lacey CJ, Bolam JP, Magill PJ (2007) Novel and distinct operational principles of intralaminar thalamic neurons and their striatal projections. J Neurosci 27:4374-4384. CrossRef Medline

Landisman CE, Long MA, Beierlein M, Deans MR, Paul DL, Connors BW
(2002) Electrical synapses in the thalamic reticular nucleus. J Neurosci 22:1002-1009. Medline

Lemieux M, Chen JY, Lonjers P, Bazhenov M, Timofeev I (2014) The impact of cortical deafferentation on the neocortical slow oscillation. J Neurosci 34:5689-5703. CrossRef Medline

Li J, Guido W, Bickford ME (2003) Two distinct types of corticothalamic EPSPs and their contribution to short-term synaptic plasticity. J Neurophysiol 90:3429-3440. CrossRef Medline

Long MA, Landisman CE, Connors BW (2004) Small clusters of electrically coupled neurons generate synchronous rhythms in the thalamic reticular nucleus. J Neurosci 24:341-349. CrossRef Medline

Mallet N, Pogosyan A, Márton LF, Bolam JP, Brown P, Magill PJ (2008) Parkinsonian $\beta$ oscillations in the external globus pallidus and their relationship with subthalamic nucleus activity. J Neurosci 28:14245-14258. CrossRef Medline

Massimini M, Huber R, Ferrarelli F, Hill S, Tononi G (2004) The sleep slow oscillation as a traveling wave. J Neurosci 24:6862-6870. CrossRef Medline

Mohajerani MH, McVea DA, Fingas M, Murphy TH (2010) Mirrored bilateral slow-wave cortical activity within local circuits revealed by fast bihemispheric voltage-sensitive dye imaging in anesthetized and awake mice. J Neurosci 30:3745-3751. CrossRef Medline

Moore BR (1980) A modification of the Rayleigh Test for vector data. Biometrika 67:175-180. CrossRef

Mukovski M, Chauvette S, Timofeev I, Volgushev M (2007) Detection of active and silent states in neocortical neurons from the field potential signal during slow-wave sleep. Cereb Cortex 17:400-414. CrossRef Medline

Nir Y, Staba RJ, Andrillon T, Vyazovskiy VV, Cirelli C, Fried I, Tononi G (2011) Regional slow waves and spindles in human sleep. Neuron 70: 153-169. CrossRef Medline

Paxinos G, Franklin K (2001) The mouse brain in stereotaxic coordinates, Ed 2. New York: Academic.

Petrof I, Sherman SM (2009) Synaptic properties of the mammillary and cortical afferents to the anterodorsal thalamic nucleus in the mouse. J Neurosci 29:7815-7819. Medline

Puig MV, Ushimaru M, Kawaguchi Y (2008) Two distinct activity patterns of fast-spiking interneurons during neocortical up states. Proc Natl Acad Sci U S A 105:8428-8433. CrossRef Medline

Rasch B, Born J (2013) About sleep's role in memory. Physiol Rev 93:681766. Medline

Reichova I, Sherman SM (2004) Somatosensory corticothalamic projections: distinguishing drivers from modulators. J Neurophysiol 92:21852197. CrossRef Medline

Rovó Z, Ulbert I, Acsády L (2012) Drivers of the primate thalamus. J Neurosci 32:17894-17908. CrossRef Medline

Ruiz-Mejias M, Ciria-Suarez L, Mattia M, Sanchez-Vives MV (2011) Slow and fast rhythms generated in the cerebral cortex of the anesthetized mouse. J Neurophysiol 106:2910-2921. CrossRef Medline

Sanchez-Vives MV, McCormick DA (2000) Cellular and network mechanisms of rhythmic recurrent activity in neocortex. Nat Neurosci 3:10271034. CrossRef Medline

Sharma AV, Wolansky T, Dickson CT (2010) A comparison of sleeplike slow oscillations in the hippocampus under ketamine and urethane anesthesia. J Neurophysiol 104:932-939. CrossRef Medline

Sherman SM, Guillery RW (1996) Functional organization of thalamocortical relays. J Neurophysiol 76:1367-1395. Medline

Sherman SM, Guillery RW (1998) On the actions that one nerve cell can have on another: distinguishing "drivers" from "modulators." Proc Natl Acad Sci U S A 95:7121-7126.

Shibata H (1998) Organization of projections of rat retrosplenial cortex to the anterior thalamic nuclei. Eur J Neurosci 10:3210-3219. CrossRef Medline

Shu Y, McCormick DA (2002) Inhibitory interactions between ferret thalamic reticular neurons. J Neurophysiol 87:2571-2576. Medline

Siapas AG, Lubenov EV, Wilson MA (2005) Prefrontal phase locking to hippocampal $\theta$ oscillations. Neuron 46:141-151. CrossRef Medline

Slézia A, Hangya B, Ulbert I, Acsády L (2011) Phase advancement and nucleus-specific timing of thalamocortical activity during slow cortical oscillation. J Neurosci 31:607-617. CrossRef Medline

Steriade M, Nuñez A, Amzica F (1993) A novel slow ( $<1 \mathrm{~Hz}$ ) oscillation of 
neocortical neurons in vivo: depolarizing and hyperpolarizing components. J Neurosci 13:3252-3265. Medline

Steriade M, Timofeev I, Grenier F (2001) Natural waking and sleep states: a view from inside neocortical neurons. J Neurophysiol 85:1969-1985. Medline

Stroh A, Adelsberger H, Groh A, Rühlmann C, Fischer S, Schierloh A, Deisseroth K, Konnerth A (2013) Making waves: initiation and propagation of corticothalamic $\mathrm{Ca}^{2+}$ waves in vivo. Neuron 77:1136-1150. CrossRef Medline

Theyel BB, Llano DA, Sherman SM (2010) The corticothalamocortical circuit drives higher-order cortex in the mouse. Nat Neurosci 13:84-88. CrossRef Medline

Timofeev I, Steriade M (1996) Low-frequency rhythms in the thalamus of intact-cortex and decorticated cats. J Neurophysiol 76:4152-4168. Medline

Timofeev I, Contreras D, Steriade M (1996) Synaptic responsiveness of cortical and thalamic neurones during various phases of slow sleep oscillation in cat. J Physiol 494:265-278. Medline

Timofeev I, Grenier F, Bazhenov M, Sejnowski TJ, Steriade M (2000) Origin of slow cortical oscillations in deafferented cortical slabs. Cereb Cortex 10:1185-1199. CrossRef Medline

Timofeev I, Grenier F, Steriade M (2001) Disfacilitation and active inhibi- tion in the neocortex during the natural sleep-wake cycle: an intracellular study. Proc Natl Acad Sci U S A 98:1924-1929. CrossRef Medline

Trageser JC, Keller A (2004) Reducing the uncertainty: gating of peripheral inputs by zona incerta. J Neurosci 24:8911-8915. CrossRef Medline

Trageser JC, Burke KA, Masri R, Li Y, Sellers L, Keller A (2006) Statedependent gating of sensory inputs by zona incerta. J Neurophysiol 96: 1456-1463. CrossRef Medline

van den Heuvel MP, Sporns O (2011) Rich-club organization of the human connectome. J Neurosci 31:15775-15786. CrossRef Medline

Van der Werf YD, Witter MP, Groenewegen HJ (2002) The intralaminar and midline nuclei of the thalamus: anatomical and functional evidence for participation in processes of arousal and awareness. Brain Res Rev 39:107-140. CrossRef Medline

Van Horn SC, Sherman SM (2007) Fewer driver synapses in higher order than in first order thalamic relays. Neuroscience 146:463-470. CrossRef Medline

Veinante P, Lavallée P, Deschênes M (2000) Corticothalamic projections from layer 5 of the vibrissal barrel cortex in the rat. J Comp Neurol 424:197-204. CrossRef Medline

Volgushev M, Chauvette S, Mukovski M, Timofeev I (2006) Precise longrange synchronization of activity and silence in neocortical neurons during slow-wave sleep. J Neurosci 26:5665-5672. CrossRef Medline 